Politische Morde ohne mediale Inszenierung - das war für linke Terroristen immer ausgeschlossen. Sie wollten die Öffentlichkeit erreichen, ihre Botschaften und Heilsideologien in die Gesellschaft tragen. Gabriele Metzler von der HumboldtUniversität in Berlin zeigt, dass der Staat darauf nicht unvorbereitet war. Er reagierte auf die medialen Stilisierungen und Herausforderungen des Terrorismus nicht nur mit politischen, polizeilichen und legislativen Mitteln, sondern auch mit einer breit angelegten publizistischen und performativen Gegenstrategie, die seine Widersacher sogar im eigenen Umfeld isolierte. Besonderen Reiz gewinnt dieser methodisch innovative Ansatz durch den transatlantischen Vergleich: Handelte Washington, das mit den linksradikalen Weathermen konfrontiert war, anders als Bonn, wo man mit der RAF zu tun hatte?

Gabriele Metzler

\title{
Konfrontation und Kommunikation
}

Demokratischer Staat und linke Gewalt in der Bundesrepublik und den USA in den 1970er Jahren

„Ich werde nun eine Kriegserklärung verlesen. “1 Mit diesen Worten einer jungen Frau setzte eine Tonbandnachricht ein, die am 21. Mai 1970 veröffentlicht wurde. Zwei Wochen später, am 5. Juni 1970, einige tausend Kilometer entfernt, eine andere Nachricht. Sie stellte klar: „Es hat keinen Zweck, den falschen Leuten das Richtige zu erklären. Das haben wir lange genug gemacht. Die Aktion haben wir nicht den intellektuellen Schwätzern, den Hosenscheißern, den Alles-besser-Wissern zu erklären, sondern den potentiell revolutionären Teilen des Volkes“. Am Ende folgte dann das Programm: „Die Klassenkämpfe entfalten / Das Proletariat organisieren / Mit dem bewaffneten Widerstand beginnen / Die Rote Armee aufbauen."

Die Rote Armee Fraktion (RAF) hatte mit der „Aktion“, der Befreiung Andreas Baaders aus dem Gefängnis, ihre konstituierende Tat vollzogen. Weit entfernt von den Geschehnissen in Berlin hatte kurz zuvor eine Gruppierung in den USA in einem Kommuniqué erklärt, nun in den bewaffneten Kampf einzutreten. Sie nannte sich „Weatherman Underground“, nach einem Song von Bob Dylan, in dem es hieß: „You don't need a weatherman to know which way the wind blows.”

\footnotetext{
${ }^{1}$ Communique \#1 From the Weatherman Underground [The Berkeley Tribe, 31. Juli 1970], in: Harold Jacobs (Hrsg.), Weatherman, Berkeley 1970, S. 509-511, hier S. 509. - Bei diesem Aufsatz handelt sich um die stark erweiterte Fassung meiner Antrittsvorlesung an der HumboldtUniversität zu Berlin.

${ }^{2}$ Die Rote Armee aufbauen. Erklärung zur Befreiung Andreas Baaders vom 5. 6. 1970, in: Rote Armee Fraktion. Texte und Materialien zur Geschichte der RAF, Berlin 1997, S. 24-26, hier S. 24 u. S. 26.

${ }^{3}$ Bob Dylan, Subterranean Homesick Blues, in: Ders., Songtexte 1962-1985, Frankfurt a. M. 1987, S. 452-455; zur Namensgebung vgl. Jeremy Varon, Bringing the War Home. The Wea-
} 
Aus welcher Richtung der Wind nun wehen würde, war klar: Anders als die protestierenden Studenten von 1968, anders als die Flugblattverteiler und Barrikadenbauer von Berlin, Berkeley oder Paris, würde man nun nicht mehr nur reden und hoffen, die Verhältnisse zu verändern. Nun war die Zeit für die „revolutionäre Tat“ gekommen, die keine falschen Kompromisse mehr kannte, die die Massen mobilisieren und die saturierten westlichen Gesellschaften endlich aufrütteln würde, sie eintreten ließe in den weltweiten Kampf gegen Imperialismus und Krieg, gegen Kapitalismus und Ungerechtigkeit.

Was hier großspurig angekündigt wurde, setzte man in den folgenden Jahren mit wachsendem Zynismus in die Praxis um. Die Gesellschaften Westeuropas und der USA erlebten in den 1970er und 1980er Jahren, teils sogar noch später, eine Serie politisch motivierter Bombenanschläge, Entführungen und Morde. Der „Terror“ wurde in dieser Zeit zu einer die westlichen Gesellschaften prägenden Erscheinung, deren Effekte bis in das Alltagsleben hinein ausstrahlten und staatliches Handeln maßgeblich mit bestimmten.

Schon früh ist in der wissenschaftlichen Debatte der symbolische, der kommunikative Charakter dieser Gewalttaten hervorgehoben worden ${ }^{4}$. In der kriminologischen, soziologischen, dann auch in der historischen Forschung bürgerte sich für diese Form von Gewalt der Begriff „Terrorismus“ ein, obwohl er eigentlich ein zeitgenössischer und mithin ein Quellenbegriff ist ${ }^{5}$. Jeder Form von Gewaltausübung wohnt eine kommunikative Qualität inne, und doch unterscheidet sich politische Gewalt schon phänomenologisch von "gewöhnlicher" Gewalt. Unter politischer Gewalt verstehe ich „ein besonderes kollektives Handlungsrepertoire, das einen erheblichen Grad physischer Gewalt mit sich bringt und zu bestimmten Zeiten in der dominanten politischen Kultur als illegal angesehen wird"6. Auch die Terroristen selbst haben für sich in Anspruch genommen, nicht „gewöhnliche" Gewalttäter zu sein, wollten sie doch nicht einzelne Menschen, sondern das System, das sie repräsentierten, treffen. Unter dem Eindruck diskursanalytisch und kulturwissenschaftlich inspirierter Sichtweisen hat auch die historische Forschung, gerade in einer Reihe aktueller Arbeiten, das Symbolische immer wieder betont; bisweilen ist sie dabei von einem gewissen Zynismus nicht frei gewesen, der stets mitschwingt, wenn als bloßer symbolischer Akt gefasst wird, was Men-

ther Underground, the Red Army Faction, and Revolutionary Violence in the Sixties and Seventies, Berkeley/Los Angeles/London 2004, S. 49.

${ }^{4}$ Vgl. Walter Laqueur, Terrorismus, Kronberg/Ts. 1976; Alex P. Schmid/Janny de Graaf, Violence as Communication. Insurgent Terrorism and the Western News Media, London 1982; v.a. Peter Waldmann, Terrorismus. Provokation der Macht, 3., akt. Aufl., Hamburg 2011 [1998], bes. Kap. 1, 2 und 4.

${ }^{5}$ Obwohl „Terrorismus“ eine zeitgenössische Zuschreibung ist, verwende ich diesen Begriff in meinem Text ohne Anführungszeichen - der besseren Lesbarkeit halber, und weil er sich im Sprachgebrauch als analytische Kategorie zur Beschreibung bestimmter Formen politischer Gewalt eingebürgert hat.

${ }^{6}$ Donatella della Porta, Politische Gewalt und Terrorismus: Eine vergleichende und soziologische Perspektive, in: Klaus Weinhauer/Jörg Requate/Heinz-Gerhard Haupt (Hrsg.), Terrorismus in der Bundesrepublik. Medien, Staat und Subkulturen in den 1970er Jahren, Frankfurt a. M./New York 2006, S. 33-58, hier S. 35. 
schen in ihrer körperlichen oder psychischen Unversehrtheit verletzt oder sie gar um ihr Leben bringt.

Gleichwohl können wir die symbolischen, kommunikativen Dimensionen des Geschehens nicht ausblenden, wenn wir es verstehen und erklären wollen. Denn was die linken Gewalttaten aus der gewöhnlichen Kriminalität heraushob und sie von „gewöhnlichen“ Mordtaten unterschied, war der Sinn, der ihnen beigelegt wurde, waren die Erfahrungen und Weltsichten, die durch die Taten sichtbar wurden.

Man könnte dies als Selbstgespräche irregeleiteter politischer Autisten abtun und viele Historiker haben das auch getan -, wenn den Terroristen darauf nicht geantwortet worden wäre, nämlich von den Vertretern des Staates, dem der Krieg erklärt worden war. Diese teilten ihre eigenen Erfahrungen mit und machten ihre Weltbilder und Leitvorstellungen sichtbar. Nur dadurch wurde aus den Selbstgesprächen der Täter eine Kommunikation zwischen zwei Sprechenden, bei der obendrein ein dritter präsent war. Für die gewaltsoziologische Forschung kann aus Gewalt nämlich nur dann soziales Handeln werden, wenn ein „beobachtender Dritter" beteiligt ist ${ }^{7}$. Das können entweder Teile der Öffentlichkeit sein, die sich durch politische Gewalttaten bedroht fühlen, oder auch andere Gruppen, die durch die „Propaganda der Tat“ mobilisiert werden sollen" ${ }^{8}$.

Diese „triadische Konstruktion“9 soll den Hintergrund bilden für die folgenden Überlegungen. Am empirischen Beispiel der Bundesrepublik und der USA soll zunächst die kommunikative Dimension linker politischer Gewalt analysiert und erklärt werden, was ihren spezifischen Charakter und ihren historischen Ort ausmachte. Vieles davon ist bereits in der Forschung aufgearbeitet und beschrieben worden, weshalb dieser erste Teil kurz ausfallen kann. Im Mittelpunkt steht dabei für die deutsche Seite die RAF, für die USA die Weathermen bzw. Weatherman Underground Organization (WUO). Mehr Gewicht wird dem zweiten Teil gegeben, in dem die staatliche Kommunikation eingehender beleuchtet wird. Auf sie bezogen, sei vorab als These formuliert, dass die staatliche „Antiterrorpolitik“ in hohem Maße performativ und daher auch und gerade durch Dramatisierung und Theatralisierung, durch bestimmte Weisen zu reden und Bilder zu nutzen, markant geprägt war.

\footnotetext{
${ }^{7}$ Vgl. Jan Philipp Reemtsma, Vertrauen und Gewalt. Versuch über eine besondere Konstellation der Moderne, Hamburg 2008, zum „Dritten“ S. 471 ff.; Birgitta Nedelmann, Gewaltsoziologie am Scheideweg. Die Auseinandersetzung in der gegenwärtigen und Wege der künftigen Gewaltforschung, in: Trutz von Trotha (Hrsg.), Soziologie der Gewalt, Opladen/Wiesbaden 1997, S. 59-85.

${ }^{8}$ Vgl. Herfried Münkler, Guerillakrieg und Terrorismus. Begriffliche Unklarheit mit politischen Folgen [1992], in: Wolfgang Kraushaar (Hrsg.), Die RAF und der linke Terrorismus, 2 Bde., Hamburg 2006, Bd. 1, S. 78-102, hier S. $94 \mathrm{ff}$.

${ }^{9}$ Reemtsma, Vertrauen und Gewalt, S. 467.
} 


\section{„Bring the War Home!" - Die terroristische Herausforderung}

Weathermen, Rote Armee Fraktion wie auch viele andere Gruppen, die Gewalt proklamierten, legitimierten und ausübten - von den Roten Brigaden in Italien bis zur japanischen Roten Armee -, waren „Entmischungsprodukte“10 der 68er Protestbewegungen. Weathermen und RAF sind gut geeignete Vergleichsfälle, weil sie zum einen in etwa zeitgleich entstanden, eine ähnliche Genese aufweisen sowie hinsichtlich der sozialen Herkunft und der Geschlechterzugehörigkeit ihrer Mitglieder ähnlich strukturiert waren.

Die eingangs erwähnte Weathermen „Kriegserklärung“ vom Mai 1970 vollendete den Weg einiger linker Mitglieder der US-amerikanischen Students for a Democratic Society (SDS) in die Gewalt. Bernardine Dohrn, die junge Frau, die die Erklärung auf Tonband sprach, hatte schon im Sommer 1968 für Solidarität mit der radikalen Schwarzen-Bewegung der Black Panther Party plädiert ${ }^{11}$. Während der SDSVersammlung in Chicago im Juni 1969 spaltete sich der Studentenverband, die Radikalen, zum Kampf Bereiten scharten sich nun hinter jenen, die schon im Vorfeld des Kongresses das Weatherman-Kommuniqué in den New Left Notes platziert hatten ${ }^{12}$. Eine Zeit der Selbstfindung, aber auch der hektischen Organisation und Vorbereitung auf die „Tat“ begann. „Bring the War Home!“ - unter diesem Motto kündigten die „Revolutionäre“ schließlich an, Zehntausende von Demonstranten würden nach dem 8. Oktober 1969, dem zweiten Todestag Che Guevaras, in Chicago an „vier Tagen des Zorns“ militante Aktionen durchführen. Schließlich waren es dann aber nur einige Hundert Demonstranten, die sich, martialisch gekleidet mit Motorradstiefeln, Helmen, teils mit Gasmasken, Schlachten mit der Chicagoer Polizei lieferten. Versuche, den Prozess gegen die „Chicago Eight" zu stören, die wegen ihrer Beteiligung an den Aktionen gegen den Parteitag der Demokraten im Sommer des Vorjahres vor Gericht standen, blieben ohne Erfolg $^{13}$. Tagelang hielten die Auseinandersetzungen an, am Ende waren rund 230 Demonstranten verhaftet, zahlreiche ebenso verletzt wie einige Polizisten ${ }^{14}$. Die Massenbewegung jedoch blieb aus.

\footnotetext{
${ }^{10}$ Vgl. Wolfgang Kraushaar (im Gespräch mit Jörg Herrmann), Die Aura der Gewalt. Die „Rote Armee Fraktion“ als Entmischungsprodukt der Studentenbewegung - Erinnerungen, Interpretationen, Hypothesen, in: Lettre International 14 (2001), H. 52, S. 7-16.

${ }^{11}$ Bernardine Dohrn, White Mother Country Radicals, in: New Left Notes, 29. 7. 1968, S. 5, zit. nach Ingo Juchler, Die Weathermen, in: Kraushaar (Hrsg.), Die RAF, Bd. 2, S. 768-781, hier S. 776 .

${ }^{12}$ Vgl. Daniel Berger, Outlaws of America. The Weather Underground and the Politics of Solidarity, San Francisco 2005, S. 82 ff.; Erkärung „You Don't Need a Weatherman To Know Which Way The Wind Blows", New Left Notes, 18.6.1969, auch abgedr. in: Jacobs (Hrsg.), Weatherman, S. 51-91.

${ }^{13}$ Vgl. John Schultz, The Chicago Conspiracy Trial, überarb. Aufl., Chicago/London 2009, S. $90-105$.

${ }^{14}$ Bericht der Illinois Crime Commission, abgedr. in: The Weather Underground. Report of the Subcommittee to Investigate the Administration of the Internal Security Act and Other Internal Security Laws of the Committee of the Judiciary, United States Senate, Washington
} 
Die Weathermen zogen sich auf eine Position als heroische Avantgarde der Revolution zurück ${ }^{15}$. Die Erfahrungen, wie Demonstrationen durch die Polizei niedergeschlagen wurden, und vor allem wie eine Chicagoer Polizeieinheit im Dezember 1969 die Black Panther Fred Hampton und Mark Clark im Schlaf erschoss, radikalisierten die Weathermen weiter. Eine vorerst letzte öffentliche Kundgebung im Dezember 1969, der „SDS National War Council“ in Flint, Michigan, war eine Gedenkveranstaltung für die getöteten Panther ${ }^{16}$, zugleich diente sie den Weathermen aber auch dazu, sich auf den bewaffneten Kampf einzuschwören, der fortan im Untergrund zu führen sei ${ }^{17}$.

In ihren Kampf rekurrierten die Weathermen vornehmlich auf Bombenanschläge auf Polizeistationen und Militäreinrichtungen, Gerichte und Bürogebäude von Firmen, kleinere Anschläge verübten sie 1971/72 auch auf das Capitol und das Pentagon. Dieses im internationalen Vergleich moderate Verhältnis der Weathermen zu Gewalt und Mord als Instrumente des politischen Kampfes wurde maßgeblich geprägt durch eine einschneidende Erfahrung, als im März 1970 drei Mitglieder der Gruppe bei der Vorbereitung eines Bombenattentats in einem New Yorker Townhouse ums Leben kamen ${ }^{18}$. Danach beantworteten die Weathermen die Frage nach der Legitimität von Gewalt „gegen Sachen“ oder „gegen Menschen“ eindeutig zugunsten der ersteren. „The townhouse“, ließen sie ihre Anhänger wissen, „forever destroyed our belief that armed struggle is the only real revolutionary struggle.“ Nun wollten sie ihren Kampf für eine „revolutionäre Kultur“ im Untergrund fortsetzen ${ }^{19}$. In der Schrift „Praerie Fire“ vom Sommer 1974, die in rund 5.000 Exemplaren in der linksalternativen Szene der Buchläden und Coffee Shops kursierte, regten sie die Gründung eines legalen politischen Arms des Weather Underground an; ihre Bomben, schrieben sie, seien „bewaffnete Propaganda“, um die politische Phantasie anzuregen, keine Anstiftung zum Guerillakrieg ${ }^{20}$. Das Ende des Vietnamkrieges, der offenkundige Misserfolg der Strategie der Gewalt in den USA selbst, aber auch das sich entspannende innenpolitische Klima der Zeit nach Nixon führten dazu, dass in den folgenden Jahren die wichtigsten Köpfe der Weathermen sich der Polizei stellten. Sie wurden fast ausnahmslos, auch aufgrund von Ermittlungspannen und als illegal erachteter Ermittlungsmethoden, zu milden Strafen verurteilt.

D.C. 1975, S.15f. Vgl. auch Berger, Outlaws, S.109-114; Varon, Bringing the War Home, S. $74-112$.

${ }^{15}$ Vgl. Juchler, Weathermen, in: Kraushaar (Hrsg.), Die RAF, Bd. 2, S. 778.

${ }^{16}$ Die „Theatralität” des „Kriegsrates“ betont auch Berger, Outlaws, S. 123.

${ }^{17}$ Vgl. ebenda, S. $118 \mathrm{ff}$.; vgl. auch den Bericht „Stormy Weather“ in: Jacobs (Hrsg.), Weatherman, S. 341-350.

${ }^{18}$ Vgl. Varon, Bringing the War Home, S. 195.

${ }^{19}$ Erklärung „New Morning - Changing Weather“, 6.12.1970, in: Bernardine Dohrn/Bill Ayers/Jeff Jones (Hrsg.), Sing a Battle Song. The Revolutionary Poetry, Statements, and Communiqués of the Weather Underground 1970-1974, New York u.a. 2006, S. 161-169, hier S. 162 u. S. 164.

${ }^{20}$ Vgl. Weather Underground Organization, Prairie Fire. The Politics of Revolutionary AntiImperialism, San Francisco 1974, abgedr. auch in: Dohrn/Ayers/Jones (Hrsg.), Sing a Battle Song, S. 231-383; Varon, Bringing the War Home, S. $291 \mathrm{ff}$. 


\section{RAF, Weathermen und die kommunikativen Dimensionen des „bewaff- neten Kampfes"}

An Ausstieg war bei den Terroristen der RAF bis zum „deutschen Herbst“ 1977 kaum zu denken. Der entscheidende Schritt in die Illegalität war im Frühsommer 1970 die Befreiung von Andreas Baader gewesen, der wenige Monate zuvor wegen Brandstiftung in zwei Frankfurter Kaufhäusern wie Gudrun Ensslin und Thorwald Proll zu einer dreijährigen Zuchthausstrafe verurteilt worden war ${ }^{21}$. Zum Zeitpunkt der Kaufhausbrände hatte auch die westdeutsche 68er-Bewegung eine intensive Debatte darüber hinter sich, ob man allein auf die Kraft der Worte und die Überzeugungsmacht von Protest auf den Straßen setzen sollte oder ob nicht der Griff zur Waffe der nächste, zwingende Schritt sein müsse, wollte man die Verhältnisse wirklich verändern ${ }^{22}$. Diejenigen, die Baader befreiten, hatten diese Frage beantwortet. In einem palästinensischen Camp ließ sich diese Gruppe in die Techniken des Guerillakampfes einweisen; nach ihrer Rückkehr nach Deutschland folgten Banküberfälle, Autodiebstähle, Einbrüche, Urkundenfälschungen. Waffen und Munition wurden gesammelt, geheime Sprengstoffdepots angelegt. Eine erste Reihe von Bombenanschlägen hatten sie in der sogenannten „Mai-Offensive“ 1972 bereits durchgeführt, als Baader, kurz danach auch die vor dem Urteil der Revisionsinstanz in Sachen Brandstiftung untergetauchte Ensslin, Meinhof und andere RAF-Mitglieder im Sommer 1972 verhaftet wurden.

Die Diskussionen über ihre Haftbedingungen, die mit großem Aufwand betriebenen Prozesse im eigens dafür errichteten Hochsicherheitstrakt von Stuttgart-Stammheim und andere Faktoren erzeugten eine zweite Welle terroristischer Gewalt, deren Ziel bald vornehmlich die Befreiung der inhaftierten Genossen war. Im Herbst 1977 fand der Terrorismus seinen Höhepunkt mit den Morden an Generalbundesanwalt Siegfried Buback, dem Bankier Jürgen Ponto und schließlich der Entführung und Ermordung des Arbeitgeberpräsidenten Hanns Martin Schleyer ${ }^{23}$.

Inwiefern lassen sich diese Taten als kommunikative Akte verstehen? Die Terroristen selbst versahen ihre Taten mit Deutungen, die sie der Öffentlichkeit mitteilten. Ohne das Interesse der Medien und ohne jene dem Terrorismus in der Regel immanenten Medialisierungsstrategien sind diese Mitteilungen nicht denkbar ${ }^{24}$.

${ }^{21}$ Vgl. Sara Hakemi /Thomas Hecken, Die Warenhausbrandstifter, in: Kraushaar (Hrsg.), Die RAF, Bd. 1, S. 316-331.

${ }^{22}$ Vgl. Wolfgang Kraushaar, Entschlossenheit: Dezisionismus als Denkfigur. Von der antiautoritären Bewegung zum bewaffneten Kampf, in: Ebenda, S. 140-156.

${ }^{23}$ Vgl. Tobias Wunschik, Baader-Meinhofs Kinder. Die zweite Generation der RAF, Opladen 1997; ders., Aufstieg und Zerfall. Die zweite Generation der RAF, in: Kraushaar (Hrsg.), Die RAF, Bd. 1, S. 472-488; Klaus Pflieger, Die Aktion „Spindy“. Die Entführung des Arbeitgeberpräsidenten Dr. Hanns-Martin Schleyer, Baden-Baden 1997.

${ }^{24}$ Für die RAF ist dies bereits sehr gut untersucht worden; vgl. Andreas Elter, Propaganda der Tat. Die RAF und die Medien, Frankfurt a. M. 2008; ders., Die RAF und die Medien. Ein Fallbeispiel für terroristische Kommunikation, in: Kraushaar (Hrsg.), Die RAF, Bd. 2, S. 10601074; Hanno Balz, Von Terroristen, Sympathisanten und dem starken Staat. Die öffentliche Debatte über die RAF in den 70er Jahren, Frankfurt a. M. 2008. 
Die Tat selbst, dann aber auch die Bekennerschreiben nach Anschlägen, theoretische Traktate über den Sinn und die historische Wichtigkeit der Gewalttat, flackernde, unscharfe Videoaufnahmen von Entführungsopfern - all das waren Botschaften, die direkt an Staat und Öffentlichkeit gerichtet waren. Dabei verwiesen sie zunächst auf die instrumentelle Bedeutung der Gewalt. Ziel der Terroristen war, den Imperialismus im allgemeinen zu bekämpfen und ein Ende des Vietnamkrieges im besonderen herbeizuführen; ihre Vorbilder waren die Befreiungsbewegungen in Lateinamerika, ihre Helden die Tupamaros in Uruguay und Castros Kämpfer auf $\mathrm{Kuba}^{25}$. Die heiligen Schriften stammten von $\mathrm{MaO}^{26}$, vor allem aber von Régis Debray ${ }^{27}$, der als Kampfgefährte Che Guevaras von der Revolution in Lateinamerika schrieb, von Ernesto Che Guevara selbst, der in etlichen Schriften erklärte, was ein Guerrillero war ${ }^{28}$, und von dem brasilianischen Schriftsteller und Revolutionär Carlos Marighella, der mit dem „Minihandbuch des Stadtguerillero“ den Guerillakampf in die Metropolen transponierte ${ }^{29}$. Daran angelehnt, verhieß das „Konzept Stadtguerilla“ auch einen Erfolg der Dschungeltaktik in den westlichen Großstädten. Anschläge auf Einrichtungen des Staates, der Polizei und des Militärs sollten das Vertrauen der Bürger in die Macht staatlicher Institutionen erschüttern ${ }^{30}$. Weathermen wie RAF bekannten sich zur Sache der unterdrückten Völker überall auf der Welt, vor allem aber in Vietnam, und sie zogen die Konfliktlinien imperialistischer Unterdrückung bis ins eigene Land hinein, wenn sie, wie die RAF, ihr Gründungskommuniqué mit dem Bild eines schwarzen Panthers versahen $^{31}$ oder sich mit der schwarzen Bürgerrechtsbewegung und besonders den militanten Black Panthers solidarisierten ${ }^{32}$.

${ }^{25}$ Vgl. Ingo Juchler, Die Studentenbewegungen in den Vereinigten Staaten und der Bundesrepublik Deutschland der sechziger Jahre. Eine Untersuchung hinsichtlich ihrer Beeinflussung durch Befreiungsbewegungen und -theorien aus der Dritten Welt, Berlin 1996; ders., Trikontinentale und Studentenbewegung. Antiimperialismus als Schibboleth, in: Kraushaar (Hrsg.), Die RAF, Bd. 1, S. 205-217; Martin Klimke/Wilfried Mausbach, Auf der äußeren Linie der Befreiungskriege. Die RAF und der Vietnamkonflikt, in: Ebenda, S. 620-643.

${ }^{26}$ Vgl. v.a. Mao Tse-tung, Theorie des Guerillakrieges oder Strategie der Dritten Welt, Reinbek 1966.

${ }^{27}$ Vgl. va. Régis Debray, Revolution in der Revolution? Bewaffneter Kampf und politischer Kampf in Lateinamerika, München 1967; vgl. auch Bernhard Gierds, Che Guevara, Régis Debray und die Focus-Theorie, in: Kraushaar (Hrsg.), Die RAF, Bd. 1, S. 182-204.

${ }^{28}$ Vgl. Ernesto Che Guevara, Was ist ein Guerillero?, in: Ders., Guerilla - Theorie und Methode. Sämtliche Schriften zur Guerillamethode, zur revolutionären Strategie und zur Figur des Guerilleros, hrsg. von Horst Kurnitzky, Berlin 1968; vgl. auch Gierds, Che Guevara, in: Kraushaar (Hrsg.), Die RAF, Bd. 1.

${ }^{29}$ Vgl. Carlos Marighella, Minihandbuch des Stadtguerilleros, o.O., o.D. [Nachdr. 1972].

${ }^{30}$ Vgl. Das Konzept Stadtguerilla, April 1971, in: Rote Armee Fraktion. Texte und Materialien, S. 27-48, hier S. 48.

${ }^{31}$ Die Rote Armee aufbauen, in: Agit 883, Nr. 62, 5.6.1970, S. 6. Im Faksimile online verfügbar unter URL:<www. http:/ / plakat.nadir.org/883/index.html> (14.6. 2011).

${ }^{32}$ Vgl. Martin Klimke, The Other Alliance. Student Protest in West Germany \& the United States in the Global Sixties, Princeton 2010, S. 126-134; Varon, Bringing the War Home, S. 204f.; zum schwierigen Verhältnis der Weathermen zu den Panthers vgl. ebenda, S. 154-165 u. S. 184-186. 
Freilich waren der Vietcong und die Tupamaros und all die anderen revolutionären Bewegungen nicht nur Vorbilder; sie waren auch Genossen in ein und demselben Kampf, den die „Kämpfer“ im Westen nach maoistischem Verständnis nun auf der „äußeren Linie“ führten ${ }^{33}$. Im Moment des Kampfes näherten sich die Kämpfer emotional, affirmativ an ihre Genossen an, wie weit entfernt diese auch sein mochten: Schon in der Vorstellung von Straßenkämpfen mit der Polizei veränderten sich die Weathermen, wie einer aus der Gruppe schrieb: „we began to feel the Vietnamese in ourselves“34. Ähnliche Identifikationen waren auf Seiten der RAF zu beobachten ${ }^{35}$.

Hier wurde eine soziale Ordnung imaginiert, in der es keine Grenzen gab. Die ganze Welt befand sich in einem Befreiungskampf, und die Kämpfer in den Metropolen waren ein Teil dieses globalen, heroischen, nachgerade mythischen Ringens. Das Subjekt der Revolution waren nicht, wie es das alte marxistische Skript ${ }^{36}$ vorsah, die proletarischen Massen in den industriellen Gesellschaften des Westens; es waren Algerier, Kubaner, Vietnamesen und mit ihnen die Stadtguerilleros in den Metropolen - eins geworden durch das Medium der Gewalt. Diese repräsentierte die eine globale Sprache, die in der Umbruchzeit der Dekolonisierung überall verstanden würde. Wer sie nicht verstand, setzte die Zukunft der Freiheit aufs Spiel. Sollte der Kampf scheitern, so lässt sich aus den Schriften von RAF und Weathermen sowie anderer Gruppierungen lesen, würden Autoritarismus und Faschismus unweigerlich erneut die Macht an sich reißen.

Den Weathermen, die mehrheitlich aus dem weißen Mittelstand stammten, galt ihr Bekenntnis zu Revolution und Gewalt als Chance auf Katharsis. Nur durch die Erfahrung des Kampfes, die Bereitschaft, den eigenen Körper für die Befreiung unterdrückter Völker und unterdrückter sozialer Gruppen im eigenen Land einzusetzen, würden die aus dem hegemonialen weißen „Schweinesystem“ hervorgegangenen und von seiner streng hierarchischen, grundlegend ungerechten Ordnung profitierenden weißen Studenten überhaupt den Revolutionären in der Dritten Welt ebenbürtig, mit ihnen solidarisch werden können. Nur so war eine neue Gesellschaft denkbar, in der dann auch, wie die Weathermen, anders als die RAF, betonten, „aus chauvinistischen Männern Revolutionäre“ würden und die „Freiheit der Frauen“ nicht mehr beeinträchtigt sei ${ }^{37}$.

${ }^{33}$ Zit. nach Klimke/Mausbach, Auf der äußeren Linie, in: Kraushaar (Hrsg.), Die RAF, Bd. 1, S. 631.

${ }^{34}$ Shin'ya Ono, A Weatherman: You Do need a Weatherman to Know Which Way the Wind Blows, in: Jacobs (Hrsg.), Weatherman, S. 227-274, hier S. 241.

${ }^{35}$ Vgl. das Beispiel bei Klimke/Mausbach, Auf der äußeren Linie, in: Kraushaar (Hrsg.), Die RAF, Bd. 1, S. 635.

${ }^{36}$ Formulierung in Anlehnung an Jeremy Varon, Refusing to Be „Good Germans“, New Left Violence as a Global Phenomenon, in: Bulletin of the German Historical Institute Washington 43 (2009), S. 21-43, hier S.31, der vom „'traditional' revolutionary script“ schreibt.

${ }^{37}$ Erklärung „New Morning, Changing Weather“, in: Dohrn/Ayers/Jones (Hrsg.), Sing a Battle Song, S. 164f. Zur Bedeutung feministischer Diskurse bei Weathermen vgl. auch Cyrana B. Wyker, Women in wargasm: The politics of women's liberation in the Weather Underground Organization, Master thesis, Univ. of Florida 2009, URL: <http://scholarcommons.usf.edu/ etd $/ 93>[7.6 .2011]$. 
RAF und Weathermen formulierten ein radikales Gegennarrativ, mit dem sie die liberal verfassten parlamentarischen Systeme des Westens in Frage stellten und für sich selbst Legitimation zu gewinnen suchten. Gegennarrative fordern dominante Machtzuweisungen heraus, wobei ihre Subjekte Handlungsmacht (agency) für sich beanspruchen und traditionelle Positionen radikal in Zweifel ziehen ${ }^{38}$. Besonders rechtsstaatliche Praktiken konfrontierten Weathermen und RAF mit einem Gegennarrativ, indem sie die Legitimität gerichtlicher Verfahren zu unterminieren suchten, nicht vor Gericht erschienen, wenn sie geladen waren, Prozesse als Angeklagte lächerlich machten, konterkarierten oder störten, oder indem sie das Gefängnis als Ort purer Repression brandmarkten oder dort als Kriegsgefangene betrachtet werden wollten. Gefangene zu befreien, galt folgerichtig als bedeutende Tat im Kampf gegen das „Schweinesystem“, wie die RAF im Kontext der Baader-Befreiung oder die Weathermen nach der Befreiung des Drogengurus Timothy Leary erklärten ${ }^{39}$. Damit negierten sie die eingespielten Regeln von Justiz und Strafvollzug ebenso radikal wie mit ihren notorischen Selbstermächtigungen, im Namen eines vermeintlich legitimen Kampfes „Recht“ zu sprechen. Während die einen sich im Extremfall anmaßten, „die korrupte Existenz Hanns-Martin Schleyers" zu beenden ${ }^{40}$, versagten die anderen der staatlichen Strafverfolgung Legitimation und Sinn: „To General Mitchell we say: Don't look for us, Dog; We'll find you first. " 41

In diesem Gegennarrativ spielte Schuld eine zentrale Rolle. Auch hierin waren sich Weathermen und RAF ähnlich. Was in der Repräsentation der einen die Bürgerrechtsfrage, war für die anderen die NS-Vergangenheit. Die Schuld aus Sicht der amerikanischen „Revolutionäre“ lag allein schon im „Privileg der weißen Haut“ begründet, die USA markierten sie pejorativ als „Mutterland“ des Imperialismus, das weltweit an Unterdrückung und Ausbeutung schuld sei ${ }^{42}$. Die RAF setzte andere Akzente. Wie Horst Mahler, der die Kaufhausbrandstifter vor Gericht verteidigte, betonte, rebellierte hier eine junge Generation gegen ihre Eltern, „die in der „NS-Zeit das millionenfache Verbrechen geduldet und sich dadurch mitschuldig gemacht" hätten"43. Auch Norbert Elias hat in seinen Betrachtungen zum westdeutschen Terrorismus auf diesen „Schuldkomplex“ der jüngeren Generation hingewiesen, der sie zu einem „Distanzierungs- und Reinigungsritual“ geführt habe $^{44}$, und die Forschung ist ihm lange in dieser Deutung gefolgt. Jüngere Arbei-

${ }^{38}$ Vgl. Michael Bamberg, Considering counter-narratives, in: Ders./Molly Andrews (Hrsg.), Considering Counter-Narratives. Narrating, resisting, making sense, Amsterdam/Philadelphia 2004, S. 351-371.

${ }^{39}$ Communiqué \#4 From the Weather Underground, 15.9.1970, in: Jacobs (Hrsg.), Weatherman, S. 516.

${ }^{40}$ Kommuniqué der Entführer an die Zeitung Libération, 19.10.1977, zit. nach Rote Armee Fraktion. Texte und Materialien, S. 273.

${ }^{41}$ Communiqué \#3 From The Weatherman Underground, 26. 7.1970, in: Jacobs (Hrsg.), Weatherman, S.515. Gemeint war Justizminister John N. Mitchell.

${ }^{42}$ Shin'ya Ono, You Do Need a Weatherman, in: Ebenda, S. 237.

${ }^{43}$ Stefan Aust, Der Baader-Meinhof Komplex, 3., erw. Aufl., Hamburg 2008, S. 76.

${ }^{44}$ Norbert Elias, Der bundesdeutsche Terrorismus - Ausdruck eines Generationenkonflikts, in: Ders., Studien über die Deutschen. Machtkämpfe und Habitusentwicklung im 19. und 20. 
ten haben allerdings Zweifel genährt, ob im Fall der RAF eine Abgrenzung vom Nationalsozialismus wirklich handlungsleitend gewesen ist oder ob der Bezug zum „Dritten Reich“ nicht instrumentalisiert wurde. Dorothea Hauser meinte gar, der Antiamerikanismus der Gruppe habe im Hinblick auf die NS-Vergangenheit exkulpierende Effekte hervorgebracht ${ }^{45}$, wenn die RAF etwa „Auschwitz“ mit den alliierten Luftangriffen auf Dresden und Hamburg gleichsetzte ${ }^{46}$. Und in der Tat spricht einiges für diese Deutung. „Auschwitz“ bei der RAF, wie auch „Faschismus" und „Imperialismus" bei RAF und Weathermen dienten primär als markante Leitbegriffe im Gegennarrativ dieser Gruppen, die auf größtmögliche Resonanz angelegt waren ${ }^{47}$. Linke in den USA warfen den Weathermen deshalb nicht zu Unrecht "guilt-organizing“" vor ${ }^{48}$.

Insgesamt war es den „Terroristen“ diesseits wie jenseits des Atlantiks wohl weniger um eine Reinigung von der Schuld ihrer Väter zu tun, sondern sie waren überzeugt, allein durch die Bereitschaft zu und die Anwendung von Gewalt das Böse schlechthin zu bekämpfen ${ }^{49}$. Die Utopie der Moderne, ein Leben ohne Gewalt zu ermöglichen, schien sich zu verwirklichen; nur ein letzter Opfergang war dafür noch nötig. „Our humble task is to organize the apocalypse“, formulierte eine den Weathermen nahestehende radikale Zeitung diese chiliastische Weltsicht. Die „Terroristen“ selbst gaben der Erwartung Ausdruck, dass aus dem „Bankrott AmeriKKKas“ (mit drei K für Ku-Klux-Klan geschrieben) „ein neues Land und ein neues Volk hervorgehen“" werde ${ }^{50}$.

Der neue Mensch, dessen Geburt man „im Kampf“ erhoffte, war nur mit der Waffe in der Hand vorstellbar ${ }^{51}$. Das war das „Sinnangebot des Untergrunds“ ${ }^{\text {" }}$. Das Leben im Untergrund, der bewaffnete Kampf, eröffnete einzigartige Möglichkeiten, das eigene Selbst zu erfahren: Militanz, erläuterte der Weatherman

Jahrhundert, Frankfurt a. M. 1989, S. 300-389, hier S. 341.

${ }^{45}$ Vgl. Dorothea Hauser, Deutschland, Italien, Japan. Die ehemaligen Achsenmächte und der Terrorismus der 1970er Jahre, in: Kraushaar (Hrsg.), Die RAF, Bd. 2, S. 1272-1298, bes. S. $1288 \mathrm{ff}$.

${ }^{46}$ So beispielsweise in der Erklärung vom 25.5. 1972 zum Bombenanschlag auf das Hauptquartier der US-Army in Heidelberg, in: Rote Armee Fraktion. Texte und Materialien, S. $147 \mathrm{f}$.

${ }^{47}$ Zum Faschismus-Vorwurf der Weathermen: „And it's a system [in America] that has colonized whole nations within this country - the nation of black people, the nation of brown people to enslave, oppress and ultimately murder the people on whose backs this country was built. (Call it fascism)“, aus: Look At It: America 1969, New Left Notes 1969, abgedr. in: Jacobs (Hrsg.), Weatherman, S. 166-174, hier S. 167. Zur „Auschwitz“-Metaphorik der RAF vgl. Ulrike Meinhofs Vergleich des „toten Trakts“ in der Justizvollzugsanstalt Köln-Ossendorf mit der „Gaskammer“ bzw. „Auschwitz“, oder Gudrun Ensslins „Unterschied toter Trakt und Isolation: Auschwitz zu Buchenwald“, beide zit. nach Aust, Baader-Meinhof-Komplex, S. 292 f.

${ }^{48}$ Juchler, Weathermen, in: Kraushaar (Hrsg.), Die RAF, Bd. 2, S. 777.

${ }^{49}$ Ebenda, S. 781; vgl. Varon, Bringing the War Home, S. 160 u. S. 220; Aust, Baader-MeinhofKomplex, S. $274 \mathrm{ff}$.

${ }^{50}$ Berkeley Tribe, April 1970, zit. nach Varon, Refusing to Be „Good Germans”, S. 35.

${ }^{51}$ Bommi Baumann, Wie alles anfing, Berlin ${ }^{4} 2007$ [1977], S. 57.

${ }^{52}$ Herfried Münkler, Sehnsucht nach dem Ausnahmezustand. Die Faszination des Untergrunds und ihre Demontage durch die Strategie des Terrors [1983], in: Kraushaar (Hrsg.), Die RAF, Bd. 2, S. 1211-1226, hier S. 1219. 
Bill Ayers, war das Maß, an dem die eigene Lebendigkeit spürbar wurde ${ }^{53}$. Folgt man dem Plädoyer Jan Philipp Reemtsmas, „Terrorismus als Lebensform [zu verstehen] “54, lassen sich drei Bedeutungen ableiten: „Terrorismus“ als Lebensform kann erstens zur Konstruktion und Inszenierung von Männlichkeit und Weiblichkeit führen. Als Beispiel sei Andreas Baader genannt, der die Rolle des Macho, ja des Desparado ebenso gut beherrschte wie jene des Dandys und Womanizers ${ }^{55}$. Eine ähnliche Verbindung von Körperlichkeit und revolutionärer Attitüde lässt sich bei den weiblichen Mitgliedern der Weathermen beobachten. Auch sie inszenierten sich als Kämpfer, die, ausgerüstet mit Schlagwaffen, schweren Stiefeln und Helmen, als „Women's Militia“ in den „Days of Rage“ eigene Akzente zu setzen und nach außen zu zeigen suchten. Weitere Aktionen der „Women's Militia“ bezogen sich vor allem auf Schulen, wo sie ,jailbreaks“ durchführten ${ }^{56}$. Aber auch nach innen wirkte Gewalt in dieser Hinsicht kommunikativ. Nach StraBenkämpfen in Seattle resümierte Susan Stern von den Weathermen, wie durch die Erfahrung gemeinsam verübter Gewalt die Wahrnehmung von Weiblichkeit, weiblicher Solidarität, ja Liebe geprägt wurde ${ }^{57}$.

„Terrorismus“ als Lebensform hat eine zweite körperzentrierte Bedeutung, die des Opfers und Märtyrers: Das vom agilen und virilen Baader am denkbar weitesten entfernte Gegenbild ist das des ausgemergelten, von fast zweimonatigem Hungerstreik gezeichneten toten Körpers von Holger Meins. „Terrorismus“ als Lebensform schloss auch den Tod mit ein und verlieh diesem als Märtyrertod Sinn. Die Fotos von Meins' Leichnam kursierten schon kurz nach seinem Tod im November 1974 in der linken Szene und entfalteten dort eine beachtliche mobilisierende Wirkung ${ }^{58}$. Als Opfertod stilisierten auch die Weathermen den Verlust von Diana Oughton, Ted Gold und Terry Robbins, die 1970 bei der Explosion in New York ums Leben gekommen waren ${ }^{59}$.

Drittens schließlich lässt sich die These vom „Terrorismus“ als Lebensform in kommunikativer Hinsicht auf die terroristische Gruppe selbst beziehen. Die gemeinsamen Erfahrungen eines Lebens mit neuen Identitäten im Untergrund, bei

53 Zit. nach Varon, Refusing to Be „Good Germans“, S. 37.

${ }^{54}$ Reemtsma, Vertrauen und Gewalt, S. 504; vgl. ders., Was heißt „die Geschichte der RAF verstehen“?, in: Kraushaar (Hrsg.), Die RAF, Bd. 2, S. 1353-1368.

${ }^{55}$ Vgl. Karin Wieland, Andreas Baader, in: Kraushaar (Hrsg.), Die RAF, Bd. 2, S. 332-349.

${ }^{56}$ Varon, Bringing the War Home, S. 81; vgl. The Motor City 9, in: Jacobs (Hrsg.), Weatherman, S. 161 f.; Women's Milita, in: Ebenda, S. 163-165; programmatisch Kathy Wilkerson, Toward a Revolutionary Women's Militia, in: Ebenda, S.91-96; Erklärung zum War Council, Honky Tonk Women, in: Ebenda, S. 313-320.

${ }^{57}$ Vgl. Susan Stern, With the Weatherman. The Personal Journal of a Revolutionary Woman, New Brunswick 2007, S. 86.

${ }^{58}$ Vgl. Petra Terhoeven, Opferbilder - Täterbilder. Die Fotografie als Medium linksterroristischer Selbstermächtigung in Deutschland und Italien während der 70er Jahre, in: Geschichte in Wissenschaft und Unterricht 58 (2007), Nr. 7/8, S. 380-399, zu Meins S. 392f.; Charlotte Klonk, Bildterrorismus. Von Meins zu Schleyer, in: Inge Stephan/Alexandra Tacke (Hrsg.), NachBilder der RAF, Köln/Weimar/Wien 2008, S. 197-215.

${ }^{59}$ Vgl. die Nachrufe im Berkeley Tribe, abgedr. in: Jacobs (Hrsg.), Weatherman, S. 484 u. S. $489 f$. 
den Weathermen stärker als bei der RAF auch geprägt von kollektiven Drogenerfahrungen, verbanden die Einzelnen miteinander. Vor allem wurden durch die gemeinsam verübten Taten soziale Logiken wirksam, die die historische Gewaltforschung auch in anderen Zusammenhängen beobachtet hat. Gewalt wirkt als Kitt in sozialen Gruppen, deren Mitglieder in der Komplizenschaft ihre moralischen Standards verändern ${ }^{60}$. Die Bereitschaft zur Gewalttat bzw. die Anwendung von Gewalt schweißt eine Gruppe zusammen und forciert eine eindeutige Grenzziehung zwischen der Gruppe und den anderen. „We're ready to fight and die“, hieß es bei den Weathermen, „and either you're on our side or you're on the side of the pigs“61. Die Denunzierung und Entmenschlichung der anderen als „Schweine“auch bei der RAF ein völlig gebräuchlicher Begriff - erleichterte und verstärkte diese scharfe Grenzziehung. Einander der Gewalt- und Kampfbereitschaft zu versichern, miteinander in einer "Sprache der Gewissheit" ${ }^{\text {662 }}$ zu reden, in Ritualen die Anwendung von Gewalt einzuüben, schließlich die gemeinsame Tat zu begehen - das bildete die kommunikative Basis, auf welcher der enge Zusammenhalt gewaltbereiter und gewalttätiger Gruppen gründete und die einen Ausstieg so schwierig machte.

Ob ein Ausstieg aus der Gewalt gelang, hing entscheidend davon ab, wie Staat und Gesellschaft mit der terroristischen Erfahrung umgingen, welche Antworten sie den Terroristen gaben, welche Weltdeutung, welchen Sinn sie kommunizierten.

\section{Staatliches Handeln gegen den Terrorismus: Narrative, Bilder, perfor- mances}

Dass nach Straftaten wie Banküberfällen, Bombenanschlägen oder Morden Polizei und Justiz tätig werden, ist für das Selbstverständnis und die Alltagspraxis westlicher Rechtsstaaten kennzeichnend und wenig überraschend. Im Fall von Weathermen und RAF gingen die staatlichen Reaktionen jedoch weit über das hinaus, was im Falle „normaler Kriminalität“ unternommen worden wäre. Dieser, wenn man so will, Überschuss an Gegenmaßnahmen ist es, der uns primär interessieren muss, wenn wir die linke politische Gewalt der 1970er Jahre als Prozess wechselseitiger Kommunikation verstehen wollen, weil in ihm besonders deutlich wird, wie soziale Ordnungen durch Gewalt herausgefordert wurden, wie die Begegnung mit der linken Gewalt - obwohl die Zahl ihrer Opfer unter derjenigen "gewöhnlicher“ Kapitalverbrechen lag - als Ausnahmesituation und als Krise wahrgenommen und gedeutet wurde.

${ }^{60}$ Bei den Weathermen wird dies offensichtlich in ihren affirmativen Reaktionen auf die Ermordung Sharon Tates durch Charles Manson und seine Anhänger; vgl. Stormy Weather, in: Jacobs (Hrsg.), Weatherman, S. 347.

${ }^{61}$ Interview with David Gilbert, zit. nach Varon, Refusing to Be „Good Germans”, S. 36.

${ }^{62}$ Cathy Wilkerson, Flying Close to the Sun. My Life and Times as a Weatherman, New York u.a. 2007, S. 263. 
Im Unterschied zu „gewöhnlicher“ Kriminalität wurde die linke Gewalt diesseits wie jenseits des Atlantiks als Angriff auf den Staat gedeutet, und sie war auch so gemeint. Aus diesem Grunde wurden die staatlichen Akteure - Regierungen, Polizei und Justiz, Ermittlungsbehörden wie FBI und Bundeskriminalamt weitaus aktiver als in anderen Fällen. Die vorliegenden historischen Studien zur Anti-Terror-Politik in der Bundesrepublik betonen, dass sich das Politikfeld stufenweise entwickelt hat und man zwischen einer ersten, vom programmatischen Leitkonzept der „Inneren Sicherheit“ bestimmten und eher konzessionsbereiten Phase (bis zur Lorenz-Entführung und den Vorgängen in Stockholm 1975), einer Phase „unter dem Primat der Strafverfolgung“ mit dem Höhepunkt des „deutschen Herbstes“ 1977 sowie schließlich der Phase der Deeskalation unterscheiden muss $^{63}$. Für die USA fehlen vergleichbare systematische Studien ${ }^{64}$. Freilich liegen erste Arbeiten vor, die den Vergleich fruchtbar genutzt haben ${ }^{65}$. Dieser Beitrag zielt vor allem darauf ab, das Potential eines kulturgeschichtlich erweiterten politikgeschichtlichen Zugriffs auf die Frage nach dem Handeln staatlicher Akteure im Kontext linker Gewalt auszuloten und das Gesamtensemble staatlicher Politik in den 1970er Jahren in den Blick zu nehmen, was auf Kosten chronologischer Nuancierungen gehen mag.

Für die liberalen Rechtsstaaten diesseits wie jenseits des Atlantiks musste es darum gehen, das staatliche Gewaltmonopol zu behaupten und die Regeln, ja die Regelhaftigkeit des Rechtsstaats zur Geltung zu bringen; es musste darum gehen, das normative Fundament liberaler Staatlichkeit zu verteidigen und den radikalen Gegenentwurf der linken Gewalttäter abzuwehren. Denn anders als „normale Kriminalität“, die ja ebenfalls rechtliche Ordnungen herausfordert, indem sie deren Regeln verletzt, boten die linken politischen Gewalttäter alternative Ordnungs-

${ }^{63}$ Johannes Hürter, Anti-Terrorismus-Politik. Ein deutsch-italienischer Vergleich 1969-1982, in: VfZ 57 (2009), S. 329-348, hier S. 345. Zur „Politik der inneren Sicherheit“ in der BRD vgl. Stephan Scheiper, Innere Sicherheit. Politische Anti-Terror-Konzepte in der Bundesrepublik Deutschland während der 1970er Jahre, Paderborn 2010; Klaus Weinhauer, „Staat zeigen“. Die polizeiliche Bekämpfung des Terrorismus in der Bundesrepublik bis Anfang der 1980er Jahre, in: Kraushaar (Hrsg.), Die RAF, Bd. 2, S. 932-947; ders., Staatsmacht ohne Grenzen? Innere Sicherheit, „Terrorismus“-Bekämpfung und die bundesdeutsche Gesellschaft der 1970er Jahre, in: Susanne Krasmann/Jürgen Martschukat (Hrsg.), Rationalitäten der Gewalt. Staatliche Neuordnungen vom 19. bis zum 21. Jahrhundert, Bielefeld 2007, S. 215-238; ders., Zwischen „Partisanenkampf“ und „Kommissar Computer“: Polizei und Linksterrorismus in der Bundesrepublik bis Anfang der 1980er Jahre, in: Ders./Requate/Haupt (Hrsg.), Terrorismus in der Bundesrepublik, S. 244-270.

${ }^{64}$ Vgl. Varon, Bringing the War Home; David Cunningham, There's Something Happening Here. The New Left, the Klan, and FBI Counterintelligence, Berkeley 2004; James Kirkpatrick Davis, The FBI's Domestic Counterintelligence Program, New York 1992.

${ }^{65}$ Vgl. Matthias Dahlke, Demokratischer Staat und transnationaler Terrorismus: Drei Wege zur Unnachgiebigkeit in Westeuropa 1972-1975, München 2011; Beatrice de Graaf, Theater van de angst. De strijd tegen terrorisme in Nederland, Duitsland, Italie en Amerika, Amsterdam 2010; vgl. auch Hürter, Anti-Terrorismus-Politik. Einen weiteren Bogen spannt Holger Nehring, The Era of Non-Violence: ,Terrorism' and the Emergence of Conceptions of NonViolent Statehood in Western Europe, 1967-1983, in: European Review of History 14 (2007), S. 343-371. 
modelle an, die radikal anders als die bestehenden waren. Sie machten dieses Angebot auf denkbar dramatische Weise - und genauso antwortete der Staat.

Tatsächlich kommt man dem komplexen Interaktionsgefüge zwischen Gewalttätern, staatlichen Akteuren und der breiteren Öffentlichkeit nur auf die Spur, wenn man auch das staatliche Handeln in seinen performativen, also auch symbolischen und theatralischen Dimensionen entschlüsselt. Die Repräsentanten des Staates mussten den Forderungen und Weltbildern der Gewalttäter eigene Deutungen entgegensetzen, wollten sie ihre Macht und die rechtliche Ordnung behaupten. Sie mussten durch ihr Sprechen und Handeln Bedeutung erzeugen und Sinn kommunizieren, damit das Vertrauen der Bürger gewahrt blieb und die soziale Ordnung nicht durch die Herausforderung der Gewalt zerbrach.

Das Gesamtensemble der staatlichen Reaktion auf die linke Gewalt lässt sich analytisch fassen, wenn man sie in ihren performativen Aspekten entschlüsselt, zugleich aber auch die Narrative, die Sprechakte und Bilderstrategien der staatlichen Akteure ernst nimmt. Grundsätzlich gilt: Akteure bringen „Bedeutung im Augenblick des Äußerns, Aufführens und Sich-Verhaltens selbst hervor“, und zwar „stets neu in actu, im Zusammenspiel aller Beteiligten“"66. Aus dieser Perspektive müssen nicht allein „Staat“ und „Terrorismus“ als Antagonisten in den Blick genommen, sondern immer auch „Dritte“, also Mehrheitsgesellschaft und Öffentlichkeit, in die Analyse mit einbezogen werden, so dass statt des bloßen Antagonismus' zweier ein komplexes Wechselspiel zwischen mehreren Akteuren und auf mehreren Ebenen erkennbar wird. Der performanztheoretische Zugriff hat obendrein den Vorteil, dass er auch Rollenwechsel von Akteuren erkennbar machen kann; d.h. „die Öffentlichkeit“ muss keineswegs nur als Zuschauer begriffen werden, vor der staatliche Akteure und „Terroristen“ gewissermaßen ihr kommunikatives Spiel aufführen.

Schon als die linken Gewalttaten erstmals thematisiert und gedeutet wurden, wohnte dem ein theatralischer, dramatischer Moment inne, waren es doch rasch hohe Repräsentanten des Staates, die sich an die Öffentlichkeit wandten, den Ernst der Lage beschworen und die staatliche Politik gegenüber der Gewalt zur „Chefsache“ machten ${ }^{67}$. Regierungschefs übernahmen selbst das Kommando - offen sichtbar im Parlament, auf Pressekonferenzen und in Fernsehansprachen, de-

${ }^{66}$ Jürgen Martschukat/Steffen Patzold, Geschichtswissenschaft und „performative turn“. Eine Einführung in Fragestellungen, Konzepte und Literatur, in: Dies. (Hrsg.), Geschichtswissenschaft und „performative turn“. Ritual, Inszenierung und Performanz vom Mittelalter bis zur Neuzeit, Köln u.a. 2003, S. 1-31, hier S. 27. Mein Verständnis von Performanz/Performativität lehnt sich eng an das der Theaterwissenschaften an; vgl. v.a. Erika Fischer-Lichte, Performance, Inszenierung, Ritual. Zur Klärung kulturwissenschaftlicher Schlüsselbegriffe, in: Martschukat/Patzold (Hrsg.), Geschichtswissenschaft, S. 33-54; dies., Ästhetik des Performativen, Frankfurt a. M. 2004. Ich folge damit in weiten Teilen einem anderen Performanz-Begriff als de Graaf, Theater van de angst, die ihrer Analyse eher ein politikwissenschaftliches Konzept zugrunde legt und „Performativität“ als einen Maßstab der „Erfolgskontrolle“ von Anti-Terror-Politik setzt; vgl. dies./Bob de Graaff, Bringing politics back in: The introduction of the ,performative power' of counterterrorism, in: Critical Studies on Terrorism 3 (2010), S. 261-275.

${ }^{67}$ De Graaf, Theater van de angst, S. 142. 
monstrativ unsichtbar in Krisenstäben und hinter verschlossenen Türen tagenden Beratungszirkeln. Sie demonstrierten körperliches Engagement, konstant ernste Gesichtszüge sowie immer wieder physische Erschöpfung, wobei sie signalisierten, dass sie an die Grenzen menschlicher Leistungsfähigkeit gingen, um der Bedrohung Herr zu werden. Für diejenigen, die in Krisenstäben und Ermittlungsbehörden die Bekämpfung des Terrorismus zu ihrer Sache machten, wurde der Anti-Terror-Kampf deshalb in gewissem Sinne auch zu einer Lebensform. Dabei bildeten sich eigene Gruppenidentitäten aus, eigene Muster zu sprechen und unausgesprochene Gelübde zu schweigen ${ }^{68}$. Häufig mussten sich die staatlichen Akteure auch in ihrer privaten Lebensführung der neuen Lage anpassen, sich dem Diktat des Personenschutzes beugen und fremdes Personal, das ihr Leben schützen sollte, selbst in den intimen Zonen ihres familiären Lebens zulassen. Öffentlich erkennbar wurde die neue Lebensform in Polizeischutz und Wagenkolonnen, die mit hoher Geschwindigkeit durch die Straßen jagten, oder im Aufgebot der Bodyguards, die die Distanz zwischen den Beschützten und den Bürgern wachsen ließen ${ }^{69}$.

Die Anti-Terror-Akteure zeigten sich fest entschlossen, die Herausforderung anzunehmen. So betonte etwa Bundeskanzler Helmut Schmidt am Abend der Schleyer-Entführung auf beiden großen Fernsehsendern, dass „der Staat [...] darauf mit aller Härte antworten“ müsse ${ }^{70}$. Die Zeit von „Geduld und Nachsicht“ sei vorüber, erklärte der US-Präsident, nun behandle man die „,anarchistischen und kriminellen Elemente als das, was sie sind“71. Härte und Entschlossenheit avancierten zu Leitbegriffen in den öffentlichen Stellungnahmen, ja der Kampf gegen die terroristische Herausforderung wurde als eine Art Wettstreit an Willensstärke gedeutet. So erklärte der Bundeskanzler nach der Erstürmung der besetzten Stockholmer Botschaft durch Polizeikräfte 1975, „diese intellektuellen Verbrecher" seien „völlig fassungslos“ gewesen, als sie von der Ablehnung ihrer Forderungen durch die Bundesregierung gehört hätten. Denn: „Denen mußte doch mal gezeigt werden, daß es einen Willen gibt, der stärker ist als ihrer. “72

Das Wechselspiel von Sichtbarkeit und Unsichtbarkeit wiederholte sich in der polizeilichen Praxis. Einerseits liefen verdeckt vor der Öffentlichkeit (bisweilen

\footnotetext{
${ }^{68}$ Anhaltspunkte bei Scheiper, Innere Sicherheit, S. 50-132 und passim. Eine systematische Untersuchung der Sprachpolitik von Krisenstäben etc. steht noch aus, unterliegt freilich besonderen Problemen des Quellenzugangs.

${ }^{69}$ Zum Thema „Personenschutz“ bereitet Maren Richter (Universität München) derzeit eine Dissertation vor (Arbeitstitel: Terrorismus und moderner Personenschutz: die bedrohte Sicherheit - Antworten des Staates auf den Terrorismus 1970 bis 1998 in der Bundesrepublik Deutschland).

${ }^{70}$ Helmut Schmidt, ARD/ZDF, 5.9.1977, 21:30 Uhr, zit. nach Scheiper, Innere Sicherheit, S. 51 .

${ }^{71}$ Richard Nixon, Statement about legislative proposals concerning explosives, 25.3. 1970, in: John T. Woolley/Gerhard Peters, The American Presidency Project [online], Santa Barbara/ CA. <http:/ / www.presidency.ucsb.edu/ws /?pid=2925> [7.6.2011].

72 „Denen mußte es mal gezeigt werden“. Interview mit Helmut Schmidt, in: Der Spiegel vom 28.4. 1975, S. 26.
} 
auch verdeckt vor dem amerikanischen Präsidenten $)^{73}$ äußerst leistungsfähige Fahndungsmaschinen auf Hochtouren, generierten und organisierten Informationen, um den gezielten und für die Täter ganz unerwarteten Zugriff zu ermöglichen; zugleich bemühten sich FBI und Verfassungsschutz darum, UndercoverAgenten und V-Männer in die linken Gewaltgruppierungen einzuschleusen, Informationen abzuschöpfen oder die Gruppen von innen her zu zersetzen ${ }^{74}-$ wobei die Informanten eine neue Identität und mit ihr im Untergrund eine neue Lebensform annahmen ${ }^{75}$-, und verdeckt prägten sich neue Ordnungen des Wissens über soziale Zusammenhänge der Gewalt aus, wenn massenhaft Daten erhoben, zueinander in Beziehung gesetzt, wissenschaftlich gedeutet und aus ihnen Erklärungen für den Terrorismus gewonnen wurden ${ }^{76}$.

Andererseits zeigte der Staat sehr offensiv seine Präsenz im Alltag der Bürger: „Der Staat [ging] in Stellung“77. Auf besondere Sichtbarkeit setzte etwa die „Hubschrauber-Springfahndung“, die in der Bundesrepublik im Mai 1972 durchgeführt wurde: „Die ganze Republik wurde von einem Netz von Kontrollstellen überzogen. Aus der Luft stürzten sich Bereitschaftspolizisten auf harmlose Kraftfahrer, Bahnpolizei durchsuchte Züge, Wasserschutzpolizei Schiffe und Häfen, die Grenzen waren dicht."78 Großangelegte Polizeikontrollen, verstärkte Sicherung an den Grenzen, aber auch vermehrte Streifengänge und -fahrten bis hin zur Absicherung vermeintlich oder tatsächlich besonders gefährdeter Gebäude

${ }^{73}$ Vgl. die Anhörung Tom Charles Hustons, vormals Berater im Weißen Haus und Autor des „Huston Plans“ zur inneren Sicherheit: Hearings before the Senate Committee to Study Governmental Operations with Respect to Intelligence Activities, Bd. 2: Huston Plan, Washington D.C. 1976, S. 3-52.

${ }^{74}$ Vgl. Cunningham, There's Something Happening Here, Appendix A (mit quantitativen Angaben). Entsprechende Aktivitäten des Verfassungsschutzes in der Bundesrepublik sind zuletzt durch die Debatten über die Täterschaft bei der Ermordung Siegfried Bubacks ins Blickfeld auch der Forschung geraten. Erste Befunde bei Wolfgang Kraushaar, Verena Bekker und der Verfassungsschutz, Hamburg 2010. Eine soziologisch orientierte historische Untersuchung der Effekte von (tatsächlicher oder befürchteter) Unterwanderung von Gewaltgruppen durch Undercover-Agenten könnte bislang übersehene gruppendynamische Prozesse freilegen; Anhaltspunkte dafür ergeben sich aus der sozialen Bewegungs-Forschung. Vgl. David Cunningham/John Noakes, „What If She's From the FBI?“ The Effects of Covert Forms of Social Control on Social Movements, in: Matthew Deflem (Hrsg.), Surveillance and Governance: Crime Control and Beyond, Bingley 2008, S. 175-197; zeitgenössisch, mit konkreten Beispielen, Gary T. Marx, Thoughts on a Neglected Category of Social Movement Participant: The Agent Provocateur and the Informant, in: American Journal of Sociology 80 (1974), S. 402-442.

${ }^{75}$ Vgl. den anschaulichen Bericht von Cril Payne, Deep Cover. An FBI Agent Infiltrates the Radical Underground, New York 1979, zum Identitäts- und Rollenwechsel bes. S. 155 ff.

${ }^{76}$ Eine wissens- und wissenschaftsgeschichtliche Untersuchung der vielfältigen zeitgenössischen kriminologischen, soziologischen, psychologischen und rechtswissenschaftlichen Analysen des „Terrorismus“ ist ein großes Desiderat der Forschung.

77 Vgl. „Der Staat geht in Stellung“, in: Der Spiegel vom 19.9. 1977. Das Titelblatt ist illustriert mit Fotos von Schützenpanzern vor dem Kanzleramt und nächtlichen Krisensitzungen im Kanzleramt.

${ }^{78}$ Dieter Schenk, Der Chef. Horst Herold und das BKA, Hamburg 1998, S. 110; zu dieser Aktion vgl. auch Weinhauer, „Staat zeigen“, in: Kraushaar (Hrsg.), Die RAF, Bd. 2, S. 938. 
durch gepanzerte Fahrzeuge repräsentierten den Staat, der seinen eigenen politischen Handlungsraum klar markierte. In Bonn wurden in den Wochen der Schleyer-Entführung Privatwohnungen führender Politiker durch große Polizeiaufgebote, Stacheldraht und Sandsäcke gesichert, im Regierungsviertel patrouillierten Polizisten in Doppelstreifen mit Schäferhunden, Schützenpanzerwagen waren aufgefahren, Mannschaftswagen der Polizei prägten das Straßenbild - es

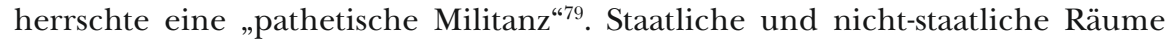
wurden auf diese Weise als getrennt voneinander gezeigt, so etwa auch, als in den USA nach einer Welle von Bombenanschlägen die Zugänge zu Polizeidienststellen durch Personen- und Taschenkontrollen stärker limitiert und kontrolliert ${ }^{80}$ oder alle Bundesbehörden rund um die Uhr von Sicherheitsdiensten bewacht wurden ${ }^{81}$.

Am prägnantesten erfolgte die Markierung getrennter Räume in den eigens für Strafprozesse gegen Gewalttäter eingerichteten Gerichtssälen. StuttgartStammheim ist hierfür gewiss das augenfälligste Beispiel, weil die Architektur hier soziales Handeln gleichsam vorstrukturierte und die Räumlichkeiten zu einem wesentlichen Teil der sozialen Interaktion wurden ${ }^{82}$. Die von Polizeisperren kontrollierte Zufahrtsstraße wie der abgesicherte Gerichtssaal lassen sich als „performative Räume“ verstehen, die das Verhältnis von Akteuren und Zuschauern bestimmten ${ }^{83}$. Die Überschreitung der Grenze vom anders strukturierten (freilich nicht minder performativen) „normalen Leben“ zur Teilnahme am Prozessgeschehen war für Angeklagte und Anwälte, aber auch für Prozessbesucher mit strapaziösen Prozeduren der Überwachung, Durchsuchung und körperlichen Kontrolle verbunden, während sich den Zuschauern am Bildschirm das Bild eines kontrollierenden, sichernden und auf rechtsstaatlichen Routinen beharrenden Staates bot.

Die Inszenierung von Sicherheit durch nimmermüde Wachsamkeit und Kontrolle suggerierte Schutz. Die Erfahrung von Staatsgewalt blieb freilich oftmals nicht abstrakt, sondern wurde für die gesuchten Gewalttäter wie im übrigen für viele Bürger auch körperlich erfahrbar, im Einsatz von Schlagstöcken und Wasserwerfern, bei Durchsuchungen nach Waffen oder Sprengstoff am Körper bis hin zur Tötung durch schießende Polizisten.

Diese Präsenz staatlicher Gewalt - im abstrakten wie in einem sehr konkreten Sinne - trug entscheidend dazu bei, dass die Zeitgenossen ihre Gegenwart vielfach als Ausnahmesituation wahrnahmen. Zu einem guten Teil war diese Art der Wahrnehmung sprachlich konstruiert. Staatliche Akteure entwarfen Narrative, die der besonderen Bedrohung gerecht werden sollten. Dazu mussten die Narra-

\footnotetext{
${ }^{79}$ Jürgen Leinemann, Schrecken Sie diese Burschen mal ab, in: Der Spiegel vom 19.9. 1977, S.32.

${ }^{80}$ Vgl. etwa den Bericht aus einem Polizeirevier im Süden Manhattans: Wade Greene, The Militants Who Play With Dynamite, in: New York Times (Magazine) vom 25. 10. 1970.

${ }^{81} \mathrm{Vgl}$. Weather Underground. Report of the Subcommittee, S. 35.

${ }^{82}$ Vgl. Balz, Von Terroristen, S. 120-177, der Stammheim im Sinne Foucaults als „Dispositiv“ deutet.

${ }^{83}$ Vgl. Fischer-Lichte, Ästhetik des Performativen, S. 188.
} 
tive plausibel sein - oder plausibilisiert werden, indem sie an ältere Erfahrungen anknüpften und neue Identitäten und Alteritäten generierten. Anders als die bisherige Forschung, die in den Äußerungen staatlicher Akteure counter-narratives erkennt ${ }^{84}$, gehe ich davon aus, dass sie nicht Gegennarrative zu etablieren, sondern vielmehr das herausgeforderte und gefährdete Master-Narrativ liberaler, rechtsstaatlich verfasster Gesellschaften zu behaupten suchten. Ihnen ging es darum, die Routinen des Rechtsstaats zu verteidigen sowie Kontinuität und Normalität wiederherzustellen ${ }^{85}$, und dazu mussten sie die Eruption linker Gewalt als einen Einbruch in diese Routinen, als außergewöhnlichen, ja als Ausnahmezustand kennzeichnen.

Einer der ersten Schritte in der sprachlichen Konstruktion des Ausnahmezustands war die Markierung einer habituellen, körperlichen Differenz zwischen „Normalgesellschaft“ und den Gewalttätern. Diese wurden als jugendliche Delinquenten beschrieben, die sich den Regeln der bürgerlichen Gesellschaft verweigerten, was bereits in ihrem Habitus und äußerem Erscheinungsbild zum Ausdruck komme. Diese Zuschreibung beanspruchte insbesondere in der frühen Phase der linken Gewalt Gültigkeit, griffen staatliche Akteure hier doch auf etablierte Muster der Charakterisierung von Anders-Sein und Devianz zurück und verwiesen immer wieder auf das „schmutzige, anti-soziale äußere Erscheinungsbild“ der linken (potentiellen) Täter ${ }^{86}$. „Alpträume von Gewalt“ sah ein amerikanischer Polizeichef aufkommen, ,als die alte weiße Furcht vor einer Invasion der Schwarzen in den Vorstädten sich verband mit der jüngeren Angst vor weiBen Verrückten, die Menschen ermordeten oder zum Krüppel machten in irgendwelchen schrecklichen Riten ihrer Sex-und-Drogen-Kulte“87. Wie das FBI, so machten auch westdeutsche Polizei und BKA auf das Äußere aufmerksam ${ }^{88}$. Diese Konstruktion von Differenz verlor indes an Plausibilität, als die Täter in den Untergrund gingen und namentlich die Mitglieder der RAF ihr äußeres Erscheinungsbild radikal verbürgerlichten. Zur Tarnung lebten „die Anarchos wie der Bürger von nebenan“89, sie trugen kurze, modische Haarschnitte und gepflegte Kleidung, was sie äußerlich nicht mehr unterscheidbar machte. Das minderte ihre potentielle Gefährlichkeit keineswegs, im Gegenteil: Nun waren tatsächliche

${ }^{84}$ Die Bedeutung der (Counter-)Narrative betont v.a. Beatrice de Graaf, Counter-Narratives and the Unrehearsed Stories Counter-Terrorists Unwittingly Produce, in: Perspectives on Terrorism 2 (2009), S. 5-11.

${ }^{85}$ Ich folge hier Bamberg, Considering counter-narratives, in: Ders./Andrews (Hrsg.), Considering Counter-Narratives, S. 360.

${ }^{86}$ Memo für FBI-Direktor, 3.6.1968, zit. nach Cunningham, There's Something Happening Here, S. 99; vgl. auch Weather Underground. Report of the Subcommittee, S. 26.

${ }^{87}$ James F. Ahern, Police in Trouble. Our Frightening Crisis in Law Enforcement, New York 1972, S. 47.

${ }^{88}$ Vgl. Weinhauer, Zwischen „Partisanenkampf“ und „Kommissar Computer“, in: Ders./Requate/Haupt (Hrsg.), Terrorismus in der Bundesrepublik, S. $257 \mathrm{f}$.

${ }^{89}$ „Eigentlich müßte jeder verdächtig sein“, in: Der Spiegel vom 12.9.1977, S.26. Vgl. auch Klaus Weinhauer, Staatsmacht ohne Grenzen?, in: Krasmann/Martschukat (Hrsg.), Rationalitäten der Gewalt, S. 223. 
oder potentielle Gewalttäter nicht mehr an ihrem Äußeren erkennbar, andere Marker mussten gesetzt werden ${ }^{90}$.

Ein zweiter Schritt bestand in der sprachlichen Identifizierung und dem labelling der Täter. Hier griff man in der Bundesrepublik wie in den USA zunächst auf Begriffe wie „Anarchist/Anarcho“ zurück, die üblich waren und mit der man an bekannte Deutungsmuster politischer Devianz anknüpfen konnte. Bald jedoch wurde die Bezeichnung „Terrorist“ geläufiger, ein „pejoratives Label“, das sowohl den damit Belegten kennzeichnet als auch diejenigen prägt, zu denen auf diese Weise gesprochen wird ${ }^{91}$. Staatliche Akteure markierten Gefährdungen durch die Terroristen als außergewöhnlich, der Superlativ war für den sprachlichen Umgang mit RAF und Weathermen reserviert. So hielt FBI-Chef J. Edgar Hoover die Weathermen für „the most violent, persistent and pernicious of revolutionary groups “92, während Repräsentanten des westdeutschen Staates die RAF ein ums andere Mal als besondere Herausforderung für die demokratische und rechtsstaatliche Ordnung bezeichneten.

In der Bundesrepublik weitaus stärker als in den USA der 1970er Jahre (heute ist das anders) schlug sich das Gefühl des Ausnahmezustands in einer Kriegs- und Bürgerkriegsmetaphorik nieder. Die Deutung des Geschehens als Krieg reflektierte einerseits die Wirkmächtigkeit von Kriegserfahrungen, sie bildete für die staatlichen Akteure andererseits aber auch ein kognitives Szenario ${ }^{93}$, in dem sie eigene biographische Erfahrungen mit aktuellen Herausforderungen verknüpfen und Orientierung gewinnen konnten. In der Ungewissheit, was um sie herum eigentlich geschah, wie gefährdet der Staat und, das sollte man nicht vergessen, wie bedroht sie selber in ihrer (physischen und politischen) Existenz tatsächlich waren, boten Kategorien wie Krieg orientierende Begriffe und handlungsleitende Vorstellungen. In diesen Kontext gehört als besonders markantes und prominentes Beispiel der drastische Vorschlag aus dem Bonner Krisenstab während der Schleyer-Entführung, ob man nicht die in Haft befindlichen Terroristen ihrerseits als Geiseln nehmen und mit ihrer Erschießung drohen sollte, wenn Schleyer nicht freikäme. Was prima vista als eine besonders bizarre Entgleisung anmutet, erweist sich auf den zweiten Blick als eine Strategie, die in der Partisanenbekämpfung im Zweiten Weltkrieg üblich war und die sich in den Erfahrungen der ehemaligen Wehrmachtsoffiziere, die nun in Bonn um das Leben Schleyers rangen,

\footnotetext{
${ }^{90}$ Diese bezogen sich dann z. B. auf Gewohnheiten wie Barzahlung von Wohnungsmieten und Stromrechnungen, wie sie der Rasterfahndung zugrunde gelegt wurden; vgl. Weinhauer, Zwischen „Partisanenkampf“ und „Kommissar Computer“, in: Ders./Requate/Haupt (Hrsg.), Terrorismus in der Bundesrepublik, S. $257 \mathrm{f}$.

${ }^{91}$ H.H.A. Cooper, What is a terrorist: a psychological perspective, in: Legal Medical Quarterly 1 (1977), S. 16-32, hier S. 16.

92 Zit. nach Weather Underground. Report of the Subcommittee, S. 38.

${ }^{93}$ Vgl. Andreas Musolff, Bürgerkriegs-Szenarios und ihre Folgen. Die Terrorismusdebatte in der Bundesrepublik 1970-1993, in: Kraushaar (Hrsg.), Die RAF, Bd. 2, S. 1171-1184, hier S. 1173; ders., Krieg gegen die Öffentlichkeit. Terrorismus und politischer Sprachgebrauch, Opladen 1996; die Kriegserfahrung thematisieren auch Scheiper, Innere Sicherheit, S. 117, und Balz, Von Terroristen, S. 286-293.
} 
festgesetzt hatte ${ }^{94}$. Der Vorschlag wurde schließlich fallen gelassen, er lässt aber erkennen, dass spezifische ältere Erfahrungen ebenso darüber mit bestimmten, was in der kritischen Situation für denkbar und sagbar gehalten wurde, wie andere jüngere Erfahrungsschichten, jene der Demokratisierung und der Durchsetzung liberaler Rechtsstaatlichkeit, die es den Akteuren nahe legten, was als machbar gelten konnte und worüber man öffentlich besser schwieg ${ }^{95}$.

Die staatlichen Akteure in den USA schöpften aus einem anderen Erfahrungsreservoir. Dort wurden die Erfahrungen des Red Scare und des Antikommunismus des frühen Kalten Krieges abgerufen, die nahtlos mit der gegenwärtigen Herausforderung durch die New Left verbunden wurden. Nicht von ungefähr stellte das FBI sein Vorgehen gegen die Radikalen auf dem College-Campus oder auf der Straße ausdrücklich unter das Vorzeichen eines Counterintelligence Program (COINTELPRO), das in den 1950er Jahren gegen die Communist Party gerichtet gewesen war und dessen Wurzeln in den Antikommunismus der ersten Nachkriegszeit nach 1918 zurückreichten ${ }^{96}$. Personelle Kontinuitäten, besonders in Gestalt von Hoover, trugen dazu bei, diese alte Deutungswelt zu perpetuieren. Damit die radikale Neue Linke und mit ihr die Weathermen in diesen Rahmen passten, wurden sie - anders als der gleichfalls vom FBI observierte Ku-Klux-Klan - zunächst nicht vorrangig nach ihrem Handeln beurteilt, sondern ihre Ideen und politischen Konzepte standen in der Ermittlungsarbeit von Hoovers Behörde im Vordergrund. Immer wurde danach gefragt, in welcher Verbindung sie zur kommunistischen Partei der USA (CPUSA) standen ${ }^{97}$.

Gerade in Nixons öffentlichen Statements und in Hoovers internen Deutungen spiegelte sich nachgerade McCarthysche Paranoia wider. In der Konstruktion des Feindes verbanden sie Antikommunismus mit der Furcht wenn nicht vor einer Invasion, so doch vor einer Unterwanderung von außen, ganz gleich, ob nun „Moskau, Peking oder Havanna die Finger im Spiel haben“ mochte ${ }^{98}$. Im kognitiven Szenario der amerikanischen Terrorbekämpfer spielten die Kontakte der Weathermen nach Kuba, Vietnam und China eine herausragende Rolle ${ }^{99}$, die Reisen der „Venceremos-Brigaden“ auf Castros Karibik-Insel wurden in der öffentlichen

${ }^{94}$ So auch gedeutet von Scheiper, Innere Sicherheit, S. 117.

${ }^{95}$ Vgl. die Analyse der Krisenstabsarbeit und der „Krisenstäbler“, in: Ebenda, S. 54 ff.

${ }^{96}$ Zur Tradition der antikommunistischen Counterintelligence vgl. Ward Churchill/Jim Vander Wall, The COINTELPRO Papers. Documents from the FBI's Secret Wars Against Domestic Dissent, Boston 1990, S. 33-48; ausführlicher Athan Theoharis, Spying on Americans. Political Surveillance from Hoover to the Huston Plan, Philadelphia 1978.

${ }^{97}$ Vgl. Cunningham, There's Something Happening Here, S. 95 ff. Innerhalb des FBI wuchs in Hoovers letzten Dienstjahren der Dissens darüber, welche Bedeutung der CPUSA überhaupt noch zuzumessen sei; vgl. die abweichende Meinung von William C. Sullivan, The Bureau. My Thirty Years in Hoover's FBI, New York/London 1979, S. $203 \mathrm{f}$.

${ }^{98}$ Comments of the Chicago Office, Foreign Influence - Weather Underground, Federal Bureau of Investigation, Weatherman Underground. Summary dated 8/20/1976, Part \#1; siehe auch die FBI-Studie „Foreign Influence“, in: Ebenda.

${ }^{99}$ Vgl. den ausführlichen Bericht „Foreign Travel and Contacts with Representatives of Foreign Governments which Influenced the WUO“, in: FBI, Weatherman Underground. Zu den Kontakten der Weatherman nach Kuba u. a. vgl. Varon, Bringing the War Home, S. 52f. u. S. $179 \mathrm{f}$. 
Berichterstattung wie in internen FBI-Papieren immer wieder thematisiert und perhorresziert. Diese Meinungsbilder fügten sich nahtlos in Nixons Konzept der „imperial presidency“, die es zu sichern galt, weil sie allein die Gewähr dafür zu bieten schien, dass die Vereinigten Staaten die Gefahren abwehrten, auf die der Präsident und sein Umfeld, verschwörungstheoretisch untermauert, verwiesen ${ }^{100}$.

Aus solchen Deutungen ließen sich in jedem Fall dichotomische Weltsichten ableiten, und eine wichtige Konsequenz daraus war die Gesetzgebung. Die Verabschiedung neuer Gesetze, die dem Staat in den 1970er Jahren erweiterte Befugnisse zur Terrorbekämpfung einräumten, ist nicht allein aus der traditionellen Praxis von Rechtsstaaten zu erklären, deren Handeln an Recht und Gesetz gebunden ist. Vielmehr vollzogen sich hier - wie im übrigen auch in der Einbeziehung der Opposition in den Bonner Krisenstab 1977 - eminent symbolische Akte, die die Handlungsfähigkeit des Staates unter Beweis stellen und diesen gegen die Markierung als präfaschistisch oder kryptofaschistisch, wie von Seiten der Terroristen kommuniziert, imprägnieren sollten. Gesetzgebung fungierte gewissermaBen als Korrektiv zum imaginierten Ausnahmezustand, der nur noch die Macht der Exekutive kannte. In der Bundesrepublik legte man größeren Wert auf Gesetzeskonformität, das korrekte Handeln des Rechtsstaates, der selbst im Moment höchster Bedrohung noch regelkonform agierte, wurde offensiver inszeniert als in den USA. Wenn besondere Umstände rasches Handeln erforderlich machten, konnten staatliche Eingriffe auch nachträglich legalisiert werden - und sie wurden es auch, und sei es in einer „beispiellose[n] legislative[n] Blitzaktion“101 wie beim sogenannten „Kontaktsperregesetz“, das innerhalb von drei Tagen Bundestag und Bundesrat passierte. Auch in dieser besonderen Situation, so betonte Bundesjustizminister Hans-Jochen Vogel, bedurften staatliche Maßnahmen nicht nur der Deckung durch Gesetze, sondern einer breiten legislativen Basis ${ }^{102}$.

Während in den deutschen Debatten die Abwehr einer Wiederkehr Weimars im Mittelpunkt stand ${ }^{103}$ und Vertrauen durch Verfahren gestiftet werden sollte, lag der rhetorische Fluchtpunkt der amerikanischen Gesetzgebung in der Sicherung der Tradition der Freiheit. Dazu zählte auch, das sensible Machtgefüge zwischen Washington und den Bundesstaaten zu wahren, deren weites Verständnis von Eigenständigkeit sich in der praktischen Fahndungs- und Polizeiarbeit bisweilen als hinderlich erwies. Aufgefangen und kompensiert wurden diese Probleme durch eine ausgeprägt föderal gefärbte Rhetorik. Schließlich brachte die amerikanische Gesetzgebung zum Ausdruck, dass die gesamten Vereinigten Staaten und nicht bloß einzelne Bundesstaaten oder Kommunen in ihrer Existenz bedroht waren,

${ }^{100} \mathrm{Zu}$ Nixon vgl. Arthur M. Schlesinger Jr., The Imperial Presidency, New York 2004 [1973], S. 255 f.; Kathryn S. Olmsted, Real Enemies. Conspiracy Theories and American Democracy, World War I to 9/11, Oxford 2009, S. $149 \mathrm{ff}$. (Kap. 5).

${ }^{101}$ Hürter, Anti-Terrorismus-Politik, S. 338.

102 Vgl. Hans-Jochen Vogel in der dritten Lesung, Deutscher Bundestag, Plenarprotokoll VIII/44. Sitzung, 29.9.1977, S. 3370.

103 Vgl. Scheiper, Innere Sicherheit, S. 141 ff.; er verweist (S. 150 ff.) darauf, dass „Weimar“ auch als positive Referenz gelten konnte (am Beispiel der sozialdemokratischen Aktualisierung Gustav Radbruchs und des Republikschutzgesetzes). 
was wiederum die Notwendigkeit eines starken, nach innen stabilen und nach außen abgeschlossenen Territorialstaats zu bekräftigen schien.

Die neuen Gesetze waren nicht nur eine Antwort auf den Ausnahmezustand, sondern trugen zugleich dazu bei, die Grenzen zwischen Gewalt und Nicht-Gewalt, zwischen „Terroristen“ und „Nicht-Terroristen“ präziser zu ziehen. Deutlichstes und in der Forschung mittlerweile breit diskutiertes Beispiel ist der in der Bundesrepublik 1976 ins Strafgesetzbuch eingefügte $§ 129 a$, der den neuen Straftatbestand der Bildung einer terroristischen Vereinigung schuf ${ }^{104}$. Strafbar war nun, eine Vereinigung zu gründen oder einer solchen beizutreten, deren Zweck es ist, Straftaten wie Morde oder Entführungen zu begehen. Das bedeutet, nicht erst die begangene Tat wird strafwürdig, sondern bereits die Mitgliedschaft in einer Gruppe, die solche Straftaten zu begehen beabsichtigte. Damit wurde die Grenzlinie zwischen Tätern und Nicht-Tätern verschoben: Als „Terrorist“ galt nun auch, wer den Finger nicht am Abzug hatte.

Eine weitere in gleiche Richtung weisende Grenzziehung erfolgte in den Diskursen über Sympathisanten, die die innenpolitische Debatte in der Bundesrepublik, in schwächerer Form aber auch in den USA, während der 1970er Jahre polarisierten ${ }^{105}$. Die öffentliche Markierung derjenigen, die Terroristen Unterschlupf boten oder Sympathien, vielleicht auch nur Verständnis für ihr Handeln äußerten, als quasi-Mittäter mag man als Hysterie der Zeit abtun, was sie vielleicht auch nur war. Vielleicht wird aber gerade hierin die Leitvorstellung einer sozialen Ordnung erkennbar, in der Zugehörigkeit und Nicht-Zugehörigkeit eindeutig zu unterscheiden sein sollten. Zu diesem Zweck wurden Entscheidungssituationen kreiert, für die es nur ein klares „Ja“ oder ein klares „Nein“ würde geben können. Solche Situationen waren selbst durch Sprachregeln bestimmt; so machte es einen großen Unterschied, ob man (Baader-Meinhof-) „Bande“ oder „Gruppe“ sagte ${ }^{106}$. Nebenbei bemerkt: Man mag eine gewisse Ironie darin erkennen, dass hier das Diktum der Terroristen „Du bekämpfst die Schweine, oder du bist für sie“ einen Widerhall fand. Wenn aber beispielsweise niedersächsische Hochschullehrer in den erregten Debatten über den „Mescalero“-Artikel aufgefordert wurden, sich (nach ihrem Beamteneid) ein weiteres Mal und ausdrücklich zur demokratischen Verfassungsordnung zu bekennen ${ }^{107}$, oder wenn amerikanische Universitäten Professoren suspendierten, sobald sie Sympathien für die Weathermen oder die Panther erkennen ließen ${ }^{108}$ - dann lassen sich solche Vorgänge als Disziplinierungen lesen, die Grenzen bestätigen und Grenzziehungen durchsetzen sollten, Rollenzuschreibungen und Rollenwechsel mit eingeschlossen; denn wer unter diesen

\footnotetext{
104 Vgl. ebenda, S. 375-378.

${ }^{105}$ Vgl. Balz, Von Terroristen, S. 77 ff.; ders., Der „Sympathisanten“-Diskurs im Deutschen Herbst, in: Weinhauer/Haupt/Requate (Hrsg.), Terrorismus in der Bundesrepublik, S. 320-350.

106 So der Vorstoß des rheinland-pfälzischen Ministerpräsidenten Bernhard Vogel, als Sympathisant müsse schon gelten, „der Baader/Meinhof-Gruppe statt -Bande sagt“, in: Der Spiegel vom 19.9. 1977, S. 24 (Fall Schleyer: „Die Dramatik muß raus“).

107 Vgl. Balz, Von Terroristen, S. $106 \mathrm{f}$.

108 Vgl. Churchill/Wall, COINTELPRO, S. 198-203.
} 
Umständen Terroristen vorübergehend bei sich aufnahm, vollzog einen Rollenwechsel und wurde, performanztheoretisch gesprochen, vom Zuschauer zum KoSubjekt der Aufführung ${ }^{109}$.

Ähnliche Befunde lassen sich mit Blick auf Strafprozesse erheben. Die Verfahren der 1970er Jahre zeigten, dass eine Entscheidung für den „Terror“ irreversibel war und die Konsequenz langjähriger Haftstrafen nach sich zog. In amerikanischen Strafprozessen gegen die Weathermen, die von der historischen Forschung kaum ansatzweise aufgearbeitet sind, sahen sich in Verfahren vor einer Grand Jury selbst Zeugen mit fragwürdigen Entscheidungssituationen konfrontiert: Beriefen sie sich auf ihr Recht, die Aussage zu verweigern, mussten sie die Konsequenz einer Strafe gewärtigen. Auch das strafprozessuale Instrument der Grand Jury diente also dazu, Entscheidungs- und Unterscheidungssituationen zu schaffen, in denen Aussage oder Nicht-Aussage klärte, wer für den Staat war oder gegen ihn ${ }^{110}$.

Selbst in banalen Alltagssituationen konnte jeder an einem solchen performativen Akt der Grenzziehung beteiligt sein - etwa bei Verkehrskontrollen, wenn die einen den Kontrollpunkt passieren durften und dadurch als „unverdächtig“ markiert wurden, während andere eine strenge Überprüfung von Fahrzeug und Personen über sich ergehen lassen mussten, was sie zumindest als „potentiell verdächtig“ kennzeichnete. Ähnliches war beispielsweise bei der Durchsuchung oder Nicht-Durchsuchung studentischer Wohngemeinschaften oder von Redaktionsräumen linker Zeitschriften festzustellen.

Wenn Grenzen sichtbarer markiert wurden, eröffnete sich den Akteuren der Terrorbekämpfung auch eine Möglichkeit zur Selbstvergewisserung. In der „permanent preparedness" fanden sie ein Mittel, mit der allgegenwärtigen und alltäglichen Bedrohung, nicht zuletzt des eigenen Lebens, umzugehen. Die Bereitschaft zu töten, vor allem aber auch das Bewusstsein, im Kampf gegen die Gewalt selbst den Tod zu finden, sollte die Polizisten an den Kontrollpunkten auf den Straßen oder bei anderen polizeilichen Einsätzen auszeichnen ${ }^{111}$.

Doch auch für die Mehrheitsgesellschaft boten sich Möglichkeiten einer positiven Identitätsbildung: Richard Nixon sprach in diesem Sinne die „silent majority“ seines Landes an, und in der Bundesrepublik wurde die Formel von der „Gemeinsamkeit der Demokraten“ zum Bindemittel einer Abwehrfront gegen die linke Gewalt ${ }^{112}$. Solche Sprechformeln waren unmittelbar an die Öffentlichkeit als „Dritter“ in der Konfrontation zwischen Staat und linker Gewalt gerichtet.

${ }^{109}$ Vgl. Fischer-Lichte, Ästhetik des Performativen, S. 64 u. S. 80.

${ }^{110}$ Aus diesem Grund war die Grand Jury seit den späten 1960er Jahren Gegenstand innenund rechtspolitischer Kontroversen in den USA; vgl. Marvin E. Frankel/Gary P. Naftalis, The Grand Jury. An Institution on Trial, New York 1977; June Barbara Kress, Rise to the Challenge. Federal Grand Jury Repression, Resistance, and Reform, 1970-1973, Diss. Univ. of California, Berkeley 1978.

111 „As never before, law enforcement must be prepared to meet this deadly threat.“ J. Edgar Hoover, Law Enforcement Faces the Revolutionary-Guerilla Criminal, in: FBI Law Enforcement Bulletin 39 (1970), H. 12, S. 20-22 u. S. 28 (Zitat).

${ }^{112}$ Hürter, Anti-Terrorismus-Politik, S. 338; auf die Wirkung von „silent majority“ verweist Rick Perlstein, Nixonland. The Rise of a President and the Fracturing of America, New York 
Während in Diskursen über Sympathisanten Entscheidungssituationen für oder gegen den Staat imaginiert wurden, konnten Markierungen als Staatsfeinde auch weit drastischere und anschaulichere Formen annehmen. Nicht nur die Terroristen setzten auf die Macht der Bilder. Auch staatliche Akteure legten sich bewusst Bilderstrategien zurecht, mit denen sich kommunikative Absichten verbanden. Im Unterschied zu den Medialisierungsstrategien der Terroristen sind diese staatlichen Vorsätze von der historischen Forschung nur wenig untersucht worden, weshalb hier nur erste Überlegungen präsentiert werden können. Zum festen Bildbestand der 1970er Jahre gehören polizeiliche Fahndungsplakate bzw. Plakate der „Ten Most Wanted People“ des FBI. Es liegt nahe, in ihnen lange etablierte Instrumente der Polizeiarbeit zu erkennen, mit deren Hilfe die per Haftbefehl Gesuchten leichter aufzuspüren waren ${ }^{113}$. Aber ihre Bedeutung weist darüber hinaus. Beatrice de Graaf deutet Fahndungsplakate als Medien, die die Angst der Öffentlichkeit wecken und die Effektivität von Staatsgewalt demonstrieren sollten ${ }^{114}$. Abgesehen davon spielten sie noch eine weitere Rolle, denn die Fahndung wurde durch sie zu einer öffentlichen Angelegenheit, indem die Bevölkerung ausdrücklich um Hinweise auf die Flüchtigen gebeten wurde. Die Öffentlichkeit wurde so von der Anti-Terror-Politik „kooptiert“115, als „Dritte“ war sie als Zuschauerin und Betrachterin der Bilder, genauso aber auch als „Mit-Fahnderin“ und damit wiederum als „Ko-Subjekt“ in performanztheoretischem Sinne unmittelbar beteiligt. Zugleich demonstrierten die auf den Plakaten abgedruckten Fotos, dass die Beschuldigten identifiziert und bekannt waren, dass man es nicht mit anonymen Kräften zu tun hatte, sondern mit Tätern, die ein Gesicht hatten. Dies war ein wichtiger Schritt, durch Sichtbarmachung ${ }^{116}$ die soziale Ordnung zu restabilisieren, deren Gegner klar gekennzeichnet werden konnten und die durch gemeinsame Anstrengung und Aufmerksamkeit über kurz oder lang gefasst und ihrer gerechten Strafe zugeführt würden. In der Bildauswahl bzw. Bildmanipulation - oft wurden private Aufnahmen der Gesuchten verwendet oder ihre Konterfeis aus Gruppenbildern herausgelöst und damit ganz neu kontextualisiert - vollzog sich schließlich die Konstruktion eines bestimmten Typus von (linkem) Täter, was, wie Susanne Regener gezeigt hat, ganz in der Tradition des Verbrecherbildes stand. Die an allen öffentlichen Plätzen präsenten Plakate ermöglichten zudem eine „trophäenmäßige Machtdemonstration“117, signalisierte doch ein

2008, S. 277.

113 Vgl. in einem breiteren Kontext Jonathan Finn, Capturing the Criminal Image. From Mug Shot to Surveillance Society, Minneapolis/London 2009.

${ }^{114}$ Vgl. De Graaf, Theater van de angst, S. 142 f.

${ }^{115}$ Finn, Capturing the Criminal Image, S. 122.

${ }^{116}$ Auf den Zusammenhang von Sichtbarmachung und Verstehen von Kriminalität verweist ebenda, S. 11.

117 Susanne Regener, „Anarchistische Gewalttäter“. Zur Mediengeschichte der RAF-Plakate, in: Gerhard Paul (Hrsg.), Das Jahrhundert der Bilder. 1949 bis heute, Göttingen 2008, S. 402409, Zitat S. 406. Eine ähnliche Interpretation aus einer sehr persönlichen Perspektive gibt Julia Albrecht, in: Dies./Corinna Ponto, Patentöchter. Im Schatten der RAF - ein Dialog, Köln 2011, S. 57 f. Zur Kontinuität des Verbrecherbildes vgl. Susanne Regener, Fotografische Erfassung. Zur Geschichte medialer Konstruktionen des Kriminellen, München 1999. 
durchgestrichenes Foto, dass wieder einer der gesuchten Gewalttäter „zur Strecke gebracht" worden war.

Im Medium des Fahndungsplakats trafen zwei Motive zusammen, die Identifizierung einer minoritären Gruppe und ihre Ausgrenzung einerseits sowie die virtuelle Stiftung von Gemeinsamkeit der Mehrheitsgesellschaft andererseits. Indem staatliche Akteure die „Gemeinsamkeit der Demokraten“ oder die „silent majority“ beschworen, nachgerade rituell wiederholte Floskeln in der politischen Sprache der Zeit, verbanden sie damit gemeinschaftsstiftende Absichten und das Bemühen, Identität sowohl positiv als auch durch die Konstruktion prägnanter Alterität herzustellen.

Um zu verhindern, dass Terroristen mit der Mehrheitsgesellschaft kommunizierten, suchte der Staat sämtliche medialen Kommunikationskanäle zu kontrollieren. Nachrichtensperren sowie Richtlinien, wie mit Botschaften von Terroristen umzugehen sei, hatten den Zweck, die Wahrnehmung der Terroristen durch Dritte - sprich: die Öffentlichkeit - einzuschränken bzw. zu regulieren. Das gelang nur in Ansätzen. Denn Ethos und Neugier professioneller Journalisten, kommerzielles Interesse der Mainstream-Verleger, Autoren und Produzenten der Untergrund-Presse und alternativer Blätter wie im Übrigen auch das Nachrichtensystem inhaftierter Täter sorgten dafür, dass die kommunikativen Beziehungen nie ganz gekappt werden konnten. Das wohlige Gruseln, das in Berichten der Mainstream-Medien über Terroristen immer wieder spürbar wurde, führte allerdings anders als es sich die RAF und die Weathermen erhofft hatten, kaum zu weiteren Solidarisierungen mit den Terroristen, sondern verstärkte den Prozess der Grenzziehung noch weiter.

Besonders deutlich wird dies, wenn man die Mainstream-Medien der Zeit im Hinblick auf mögliche Pathologisierungen der „Terroristen“ analysiert. Weitgehend ungefiltert wurden etwa polizeiliche Einschätzungen kolportiert, man habe es bei den Terroristen mit einem „Haufen von Psychopathen“ zu tun, wie sich ein Chicagoer Polizeioffizier nach einem Bombenanschlag durch die Weathermen ausgedrückt hatte ${ }^{118}$. Als Versuch der Konstruktion einer Abweichung von der neurologischen Norm lässt sich auch die akribische medizinische Analyse des Gehirns der toten Ulrike Meinhof deuten, bei der man auf die Folgen einer 1962 erfolgten Hirnoperation verwies, um ihren Weg in die RAF zu erklären ${ }^{119}$.

Überaus bemerkenswert sind in diesem Zusammenhang auch geschlechterspezifische Rollenzuweisungen. So wurde über die Frauen der RAF in den Medien häufig in stark sexualisierter Form berichtet, wobei man ihr Engagement als Folge homosexueller oder von einer idealisierten Norm abweichenden heterosexuellen Neigungen deutete ${ }^{120}$. Der BILD-Zeitung war der als „Liebeskampf um Baader“

\footnotetext{
118 Zit. nach Varon, Bringing the War Home, S. 181.

119 Vgl. Balz, Von Terroristen, S. 135.

${ }^{120}$ Völlig identische Zuschreibungen einer abweichenden Sexualität und „psychosexueller Störungen“ finden sich in den FBI-Einschätzungen der von Frauen geführten Symbionese Liberation Army, die für die Entführung Patricia Hearsts verantwortlich zeichnete; vgl. Mary Elizabeth Strunk, Wanted Women. An American Obsession in the Reign of J. Edgar Hoover, Lawrence 2010, S. 118 f.
} 
gedeutete Konflikt zwischen Meinhof und Ensslin eine Schlagzeile auf der Titelseite wert, aber auch seriösere Blätter wie der Spiegel sahen Zusammenhänge zwischen emanzipatorischen Bestrebungen und „terroristischen“ Taten von Frauen - Zusammenhänge, die auch in amtlichen Verlautbarungen hergestellt wurden ${ }^{121}$. Gisela Diewald-Kerkmann hat gezeigt, dass sexualisierende und pathologisierende Deutungen auch in den Strafprozessen gegen „Terroristinnen“ dominierten ${ }^{122}$. Waffen tragende Frauen weckten in der Bundesrepublik negativ konnotierte Assoziationen mit „Flintenweibern“ und Partisaninnen. In den USA zogen die Aktivitäten der weiblichen Weathermen ebenfalls viel Aufmerksamkeit auf sich, und auch hier lassen sich sexuell konnotierte Pathologisierungen beobachten ${ }^{123}$. Sexualisierte Deutungen wiesen ebenso wie staatliche und mediale Reaktionen auf die Besetzung einer Prüfungsklasse in einer High School oder die versuchte Störung einer Tupper-Party durch Weatherwomen unverkennbar Parallelen zu älteren Hysteriediskursen auf, die ihrerseits Teil von Geschlechterordnungen waren $^{124}$. Der offizielle Bericht über die „Days of Rage“ wies ausdrücklich auf das spezifische Aktionsrepertoire von Frauen hin, die Polizisten getreten, bespuckt und gebissen hätten ${ }^{125}$. Selbst in den USA, wo das Waffentragen von Frauen eine längere Tradition hatte und das Bild der bewaffneten Frau in den vorangegangenen Jahrzehnten gesellschaftlich domestiziert und in etablierte Rollenmuster eingeschrieben worden war, sorgte die Militanz der weiblichen Weathermen und

${ }^{121}$ Vgl. Gisela Diewald-Kerkmann, Bewaffnete Frauen im Untergrund. Zum Anteil von Frauen in der RAF und der Bewegung 2. Juni, in: Kraushaar (Hrsg.), Die RAF, Bd. 2, S. 657-675; dies., „Verführt“ - „abhängig“ - „fanatisch“: Erklärungsmuster von Strafverfolgungsbehörden und Gerichten für den Weg in die Illegalität - Das Beispiel der RAF und der Bewegung 2. Juni (1971-1973), in: Weinhauer/Haupt/Requate (Hrsg.), Terrorismus in der Bundesrepublik, S. 217-243; Balz, Von Terroristen, S. 198-218. Zum Zusammenhang von Geschlecht und Terrorismus vgl. auch den Tagungsbericht von Andreas Schneider, „Ein Exzess der Befreiung der Frau“? Terrorismus, Geschlecht und Gesellschaft in den 1970er-Jahren in transnationaler und interdisziplinärer Perspektive. 28.1.2010-29.1.2010, Gießen, in: H-Soz-uKult, 11.05.2010, <http://hsozkult.geschichte.hu-berlin.de/tagungsberichte/id=3092> [25.5.2011].

122 Vgl. Gisela Diewald-Kerkmann, Frauen, Terrorismus und Justiz. Prozesse gegen weibliche Mitglieder der RAF und der Bewegung 2. Juni, Düsseldorf 2009.

${ }^{123}$ Vgl. etwa die Berichte über die „days of rage“, in denen das Verhalten von Frauen eigens herausgestrichen wird: „Cops, troops guard city“, in: Chicago Tribune vom 10.10.1969; Ronald Koziol, Radicals go on rampage, in: Chicago Tribune vom 9.10.1969. John Kifner, Guard called in Chicago as S.D.S. roams streets, in: New York Times vom 10.10.1969, betont ausdrücklich, dass Frauen bei Auflösung der Versammlung zu weinen begonnen hätten; vgl. auch „34 held as girls stage war protest“, in: Washington Post vom 5.9.1969.

${ }^{124}$ Vgl. aus der Fülle der Literatur: Gerard Wajeman, Le Maître et l'hystérique, Paris 1982; Marianne Schuller, 'Weibliche Neurose' und Identität. Zur Diskussion der Hysterie um die Jahrhundertwende, in: Dietmar Kemper / Christoph Wulf (Hrsg.), Die Wiederkehr des Körpers, Frankfurt a. M. 1982, S. 180-194; Dorion Weickmann, Rebellion der Sinne. Hysterie ein Krankheitsbild als Spiegel der Geschlechterordnung (1880-1920), Frankfurt a. M./New York 1997.

125 Vgl. Weather Underground. Report of the Subcommittee, S. 16. 
deren radikale Umdeutung des Waffentragens für revolutionäre Zwecke für Irritationen $^{126}$.

Frauen mit der Waffe in der Hand weckten nicht erst in den 1970er Jahren widersprüchliche Reaktionen zwischen Faszination und Abscheu ${ }^{127}$. Wohl nicht nur der ehemalige Präsident des Verfassungsschutzes, Günther Nollau, wertete das Engagement der Terroristinnen als „Exzess der Befreiung der Frau“128. Aus dieser Perspektive ließ sich der Terrorismus als Teil einer umfassenderen moralischen Verfallsgeschichte deuten; Repräsentanten des Staates sahen in Gewalttaten einen Ausdruck von Normenerosion, die sich in ihren Augen auch dadurch aufhalten ließ, dass man die Geschlechterbeziehungen zu renormativieren bzw. rehierarchisieren suchte.

Zur Wiederherstellung der vermeintlich gestörten Geschlechterordnung gehörte auch, dass die Polizei oder auch viele Politiker markante Normen männlichen Verhaltens zeigten. Ihre Exposition von Härte und Entschlossenheit, Tapferkeit und Opferbereitschaft verband sich mit Gesten, die der Gesellschaft Schutz und Sicherheit versprachen; die Demonstration soldatischen Pflichtbewusstseins gehörte ebenso zur öffentlichen Rolle staatlicher Akteure wie die Betonung väterlicher Sorge und Strenge, mit der sie von „verirrten jungen Leuten“ und den „kids on campus“ sprachen.

\section{Zivilisierung der Staatsgewalt? Ein Fazit}

In den historiographischen „Krisen“-Narrativen der 1970er Jahre bildet der Verweis auf die Herausforderung der westlichen Gesellschaften durch terroristische Gewalt ein zentrales Element. Allerdings bedrohte diese Gewalt, das lässt sich zumindest für die RAF in der Bundesrepublik und die Weathermen in den USA wohl sagen, nicht weite Kreise der Bürgerschaft, sondern sie zielte auf Gebäude und Einrichtungen mit Symbolcharakter sowie auf einzelne Repräsentanten des kapitalistischen Staates. Der Mordanschlag auf diese Repräsentanten - die Tötung von Begleitern, Fahrern oder Leibwächtern wurde von den Terroristen gleichsam als Kollateralschaden zynisch in Kauf genommen - war somit immer auch symbolisch aufgeladen.

Was mit einer Kriegserklärung begann, endete mit einer Niederlage. Nirgends in der westlichen Welt führte der Terrorismus zur Revolution, nirgends hat er signifikante Mobilisierungseffekte gezeitigt. Die Weathermen zogen daraus vergleichsweise früh ihre Schlüsse und beendeten den Kampf. Die RAF bombte und tötete noch bis ans Ende der 1990er Jahre weiter, auch wenn ihren Angehörigen

\footnotetext{
${ }^{126}$ Vgl. Laura Browder, Her Best Shot. Women and Guns in America, Chapel Hill 2006, zur Domestizierung im Kontrast zum Image weiblicher Gangster in der Vorkriegszeit S. 139f., zur Radikalisierung des Images durch die „Macho Mamas of Weatherman“ S. 170-177.

${ }^{127}$ Kontinuitäten in den USA seit den 1930er Jahren bei Strunk, Wanted Women; Balz, Von Terroristen, S. 212 ff., geht auf „tradierte Feindbilder“ der „Flintenweiber“ und „Partisaninnen“ ein.

128 Zit. nach „Ausbruch in Berlin: Das ist eine Riesensache“, in: Der Spiegel vom 12.7.1976, S. 21.
} 
längst nicht mehr die Weltrevolution vor Augen stand, sondern es ihnen vorrangig darum ging, Aufmerksamkeit auf ihre inhaftierten Genossen zu lenken und die Gruppe am Leben zu erhalten.

Trotzdem: Der Terror hinterließ seine Spuren in den Gesellschaften, aus denen er hervorging und die ihn erlebten. Das liegt auch und gerade an seinen kommunikativen Dimensionen. Jede Tat war zugleich ein Kommunikationsakt, der dem Staat und der Öffentlichkeit die Ziele des Terrors, die Weltsicht und den Erfahrungsbestand der Täter mitteilen sollte. Der Staat gab darauf Antworten, die eine Machtdifferenz verdeutlichten, weil dies keine Kommunikation zwischen Gleichen war: Die einen repräsentierten die Macht des Staates, die Macht der anderen Seite gründete sich auf eine prekäre Mischung aus moralisch gefärbtem Allmachtsgefühl und Welterlösungspathos, Verzweiflung und bedingungsloser Bereitschaft zur Tat.

Freilich trugen auch die polizeilichen, justiziellen oder gesetzlichen Maßnahmen gegen den Terrorismus Bedeutungen in sich, die im Handeln expliziert wurden und ihrerseits Vorstellungen sozialer Ordnungen transportierten. Der Staat zeigte sich und seine Vertreter zeigten - durch Gesetzgebung, Visualisierungen, körperliche Praktiken -, wer zu den Eigenen und wer zu den Fremden, den Anderen gehörte. Nach Jahren, in denen die westlichen Gesellschaften pluraler und differenzierter geworden waren, erlebten sie in den Auseinandersetzungen mit dem Terror nun den Versuch einer Wendung zur Eindeutigkeit, wobei zwischen Dafür sein und Dagegen sein, Freund und Feind zu unterscheiden war. Dass dabei auf der einen wie auf der anderen Seite von „Kampf“, ja von „Krieg“ gesprochen wurde, ist nicht als metaphorischer Überschwang zu deuten, sondern „Krieg“ war eine tatsächlich, körperlich und seelisch gemachte Erfahrung oder eine imaginierte Erfahrung, in jedem Falle aber eine, die handlungsleitend wurde. Mutatis mutandis lässt sich auch der Antikommunismus der US-amerikanischen Antiterrorpolitik als später Reflex einer Kriegserfahrung, nämlich der Erfahrung des frühen Kalten Krieges, deuten. Die Anti-Terror-Politik der 1970er Jahre setzte noch einmal biografisch grundierte, erfahrungsgesättigte Schwingungen in den westlichen Gesellschaften frei, Schwingungen, deren Amplituden freilich immer weniger weit ausschlugen.

Welche Bedeutung die Verbindung von Krieg und Gewalt in den Repräsentationen des Terrorismus hatte, wäre historisch systematisch erst noch zu untersuchen; eine Untersuchung, aus der wir für heute, da wir in einem „war on terrorism“ stecken, vermutlich viel lernen könnten. Für die 1970er Jahre kann man festhalten, dass die Dramatisierung durch Kriegsmetaphorik zusehends weniger verfing, ja dass sie sukzessive an Glaubwürdigkeit verlor. Für die Frage nach dem Wandel von Staatlichkeit in dieser Zeit ist dies eine ganz entscheidende Beobachtung: Der Staat musste, um seine Entschlossenheit und Fähigkeit zum Handeln zu zeigen und zu legitimieren, sich nicht mehr als Staat im Kriegs- und Ausnahmezustand präsentieren, dem dann nahezu jedes Mittel zur Abwehr einer feindlichen Bedrohung recht sein durfte. James Sheehan hat in seiner Studie über den „Kontinent der Gewalt“ die Erfahrung des Zweiten Weltkrieges als entscheidende Zäsur 
auf „Europas langem Weg zum Frieden“ bezeichnet ${ }^{129}$. In meinen Augen bewirkte erst das gleichsam imaginierte re-enactment dieser Erfahrungen in Europa bzw. der Kalten Kriegserfahrung in den USA eine Zivilisierung von Staats gewalt ${ }^{130}$. Ob diese Zivilisierung Bestand hat oder ob nicht staatliche Gewalt durch und nach diesen Erfahrungen nur transformiert, subtilisiert, weniger sichtbar gemacht wurde, ist eine Frage, die uns heute beschäftigen sollte. Die zeithistorische Forschung könnte zu unseren aktuellen Debatten viel beitragen.

${ }^{129}$ Vgl. James Sheehan, Kontinent der Gewalt. Europas langer Weg zum Frieden, München 2008, S. $222 \mathrm{ff}$.

${ }^{130}$ Von einer "Umcodierung" von Gewalt, freilich herbeigeführt durch zivilgesellschaftliche Akteure, spricht auch Nehring, Era of Non-Violence, bes. S. 356-358. 

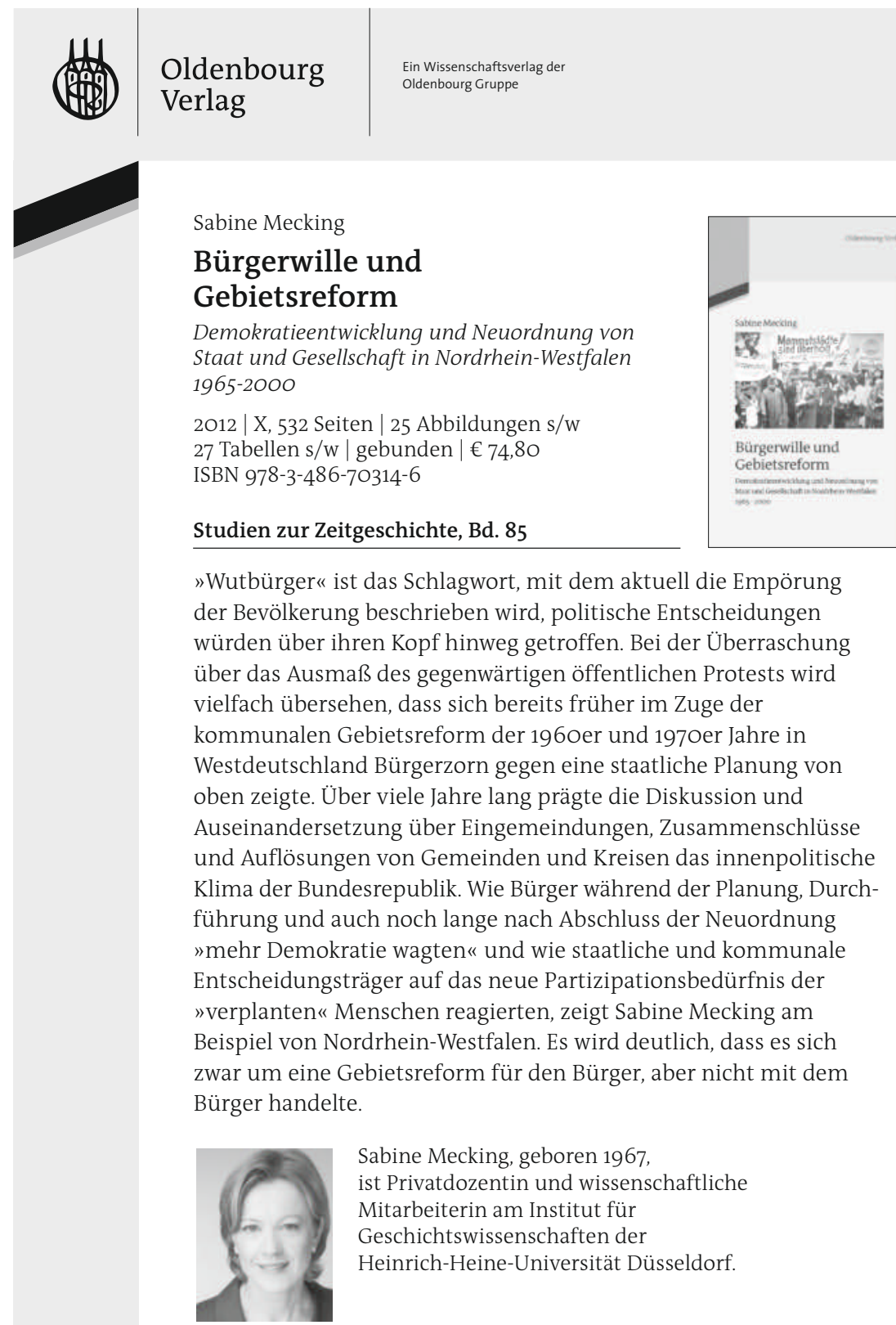

Sabine Mecking, geboren 1967 , ist Privatdozentin und wissenschaftliche Mitarbeiterin am Institut für Geschichtswissenschaften der Heinrich-Heine-Universität Düsseldorf.

Bestellen Sie in Ihrer Fachbuchhandlung oder direkt bei uns: Tel: 089/45051-248 